\title{
Metallicity Variations in the Type II Globular Cluster NGC 6934*
}

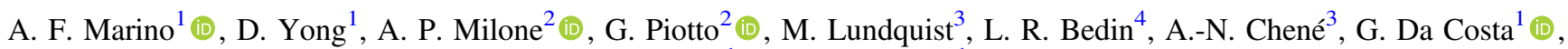 \\ M. Asplund ${ }^{1}\left(\mathbb{D}\right.$, and H. Jerjen ${ }^{1}$ (D) \\ ${ }^{1}$ Research School of Astronomy \& Astrophysics, Australian National University, Canberra, ACT 2611, Australia; anna.marino@anu.edu.au \\ ${ }^{2}$ Dipartimento di Fisica e Astronomia "Galileo Galilei"-Università di Padova, Vicolo dell’Osservatorio 3, Padova, I-35122, Italy \\ ${ }^{3}$ Gemini Observatory, Northern Operations Centre, 670 North A'ohoku Place, Hilo, HI 96720, USA \\ ${ }^{4}$ Istituto Nazionale di Astrofisica-Osservatorio Astronomico di Padova, Vicolo dell'Osservatorio 5, Padova, I-35122, Italy \\ Received 2018 January 17; revised 2018 April 9; accepted 2018 April 9; published 2018 May 25
}

\begin{abstract}
The Hubble Space Telescope photometric survey of Galactic globular clusters (GCs) has revealed a peculiar "chromosome map" for NGC 6934. In addition to a typical sequence, similar to that observed in Type I GCs, NGC 6934 displays additional stars on the red side, analogous to the anomalous Type II GCs, as defined in our previous work. We present a chemical abundance analysis of four red giants in this GC. Two stars are located on the chromosome map sequence common to all GCs, and another two lie on the additional sequence. We find (i) star-to-star Fe variations, with the two anomalous stars being enriched by $\sim 0.2$ dex. Because of our small-size sample, this difference is at the $\sim 2.5 \sigma$ level. (ii) There is no evidence for variations in the slow neutron-capture abundances over Fe, at odds with what is often observed in anomalous Type II GCs, e.g., M 22 and $\omega$ Centauri; (iii) no large variations in light elements $\mathrm{C}, \mathrm{O}$, and $\mathrm{Na}$, compatible with locations of the targets on the lower part of the chromosome map where such variations are not expected. Since the analyzed stars are homogeneous in light elements, the only way to reproduce the photometric splits on the sub-giant (SGB) and the red giant (RGB) branches is to assume that red RGB/faint SGB stars are enhanced in $[\mathrm{Fe} / \mathrm{H}]$ by $\sim 0.2$. This fact corroborates the spectroscopic evidence of a metallicity variation in NGC 6934. The observed chemical pattern resembles only partially the other Type II GCs, suggesting that NGC 6934 might belong either to a third class of GCs, or be a link between normal Type I and anomalous Type II GCs.
\end{abstract}

Key words: globular clusters: individual (NGC6934) - stars: abundances - stars: Population II

Supporting material: machine-readable table

\section{Introduction}

In the last few years there have been an increasing number of observations that indicate that a sizeable subsample of the Milky Way globular clusters (GCs) host stellar populations with different metallicities (here intended as primarily $\mathrm{Fe}$ ). These variations are in addition to the typical star-to-star variations observed for elements involved in hot- $\mathrm{H}$ burning processes, e.g., $\mathrm{C}, \mathrm{N}, \mathrm{O}, \mathrm{Na}$, and sometimes $\mathrm{Mg}$ and $\mathrm{Al}$.

The Hubble Space Telescope (HST) UV-Legacy of Galactic GCs (Piotto et al. 2015) has recently revealed that the phenonemon of multiple stellar populations in GCs is best described by the so-called "chromosome map," a diagram constructed from a combination of HST filters whose $x$ $\left(\Delta_{\mathrm{F} 275 \mathrm{~W}, \mathrm{~F} 814 \mathrm{~W}}\right)$ and $y\left(\Delta_{C}\right.$ F275W,F336W,F438W $)$ axes are mostly sensitive to helium and nitrogen, respectively (Milone et al. 2015, 2017). These diagrams were given their name because they represent a imprint of the formation processes that occurred in GCs that produced what we observe today as multiple stellar populations. The morphology of the chromosome maps varies from cluster to cluster, displaying different extensions and numbers of overdensities, corresponding to different stellar populations.

Despite some degree of variation between the maps, common features have been observed (Renzini et al. 2015;

\footnotetext{
* Based on observations with the NASA/ESA Hubble Space Telescope, obtained at the Space Telescope Science Institute, which is operated by AURA, Inc., under NASA contract NAS 5-26555. This paper includes data gathered with the $6.5 \mathrm{~m}$ Magellan Telescopes located at Las Campanas Observatory, Chile, and Gemini Telescope at Canada-France-Hawaii Telescope.
}

Milone et al. 2017). First, stars in all the GCs display a common pattern in their chromosome map extending from relatively low $\Delta_{C}$ F275W,F336W,F438W and high $\Delta_{\mathrm{F} 275 \mathrm{~W}, \mathrm{~F} 814 \mathrm{~W}}$ values to lower $\Delta_{\mathrm{F} 275 \mathrm{~W}, \mathrm{~F} 814 \mathrm{~W}}$ and higher $\Delta_{C}$ F275W,F336W,F438W. Second, a first stellar generation (1G), defined by the population of stars located at the lower $\Delta_{C}$ F275W,F336W,F438W values of the map, has been identified in all GCs investigated so far. Stars associated with the second generation (2G) are found at higher $\Delta_{C}$ F275W,F336W,F438W, defining a sequence in both $\Delta_{\mathrm{F} 275 \mathrm{~W}, \mathrm{~F} 814 \mathrm{~W}}$ and $\Delta_{C}$ F275W,F336W,F438W, that extends from lower- $\Delta_{C}$ F275W,F336W,F438W $/$ higher- $\Delta_{\mathrm{F} 275 \mathrm{~W}, \mathrm{~F} 814 \mathrm{~W}}$ toward different degrees of enhanced $\Delta_{C}$ F275W,F336W,F438W values. As these feauture have been observed as a general behavior in Milky Way GCs, the GCs showing exclusively these features have been named Type I GCs (Milone et al. 2017).

However, 10 of the $57 \mathrm{GCs}$ in the HST study have photometric peculiarities (Milone et al. 2017). These objects, named Type II GCs, in addition to the common $1 \mathrm{G}$ and $2 \mathrm{G}$ stars observed in Type I GCs, display additional sequences in the red side of their chromosome map. These red stars usually follow the same pattern as observed for the $1 \mathrm{G}$ and $2 \mathrm{G}$ stars in Type I GCs, i.e., a distribution from high- $\Delta_{\mathrm{F} 275 \mathrm{~W}, \mathrm{~F} 814 \mathrm{~W}} /$ low- $\Delta_{C}$ F275W,F336W,F438W to lower$\Delta_{\mathrm{F} 275 \mathrm{~W}, \mathrm{~F} 814 \mathrm{~W}} /$ higher- $\Delta_{C}$ F275W,F336W,F438W. However, the lowest $\Delta_{C}$ F275W,F336W,F438W values observed for the red stars change from cluster to cluster.

All the Type II GCs for which chemical abundances are available from spectroscopy have been classified as anomalous GCs, i.e., as GCs exhibiting internal variations not just in the light elements involved in hot-H burning processes, but also in the bulk 

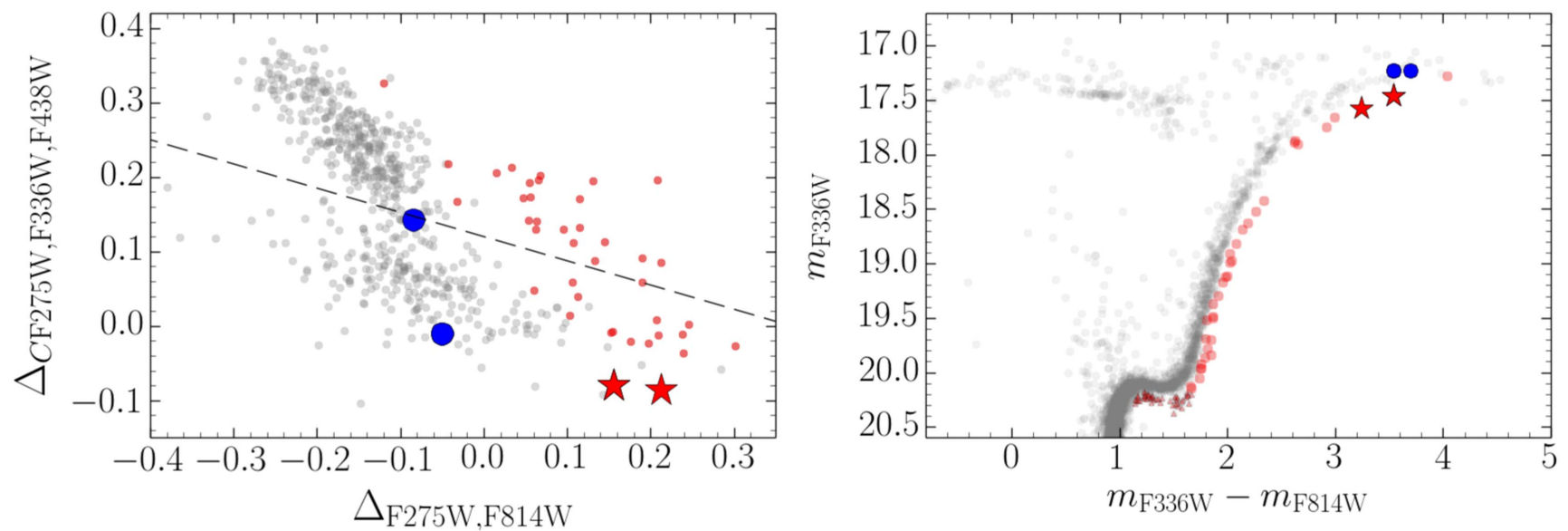

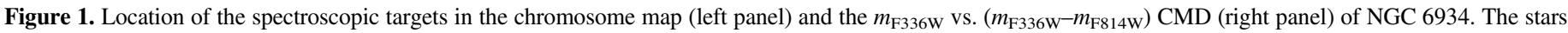

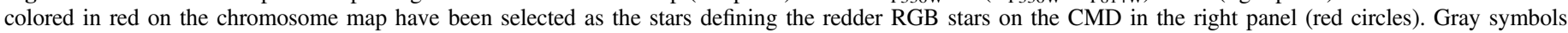

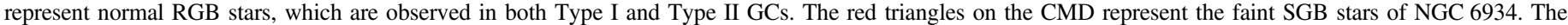

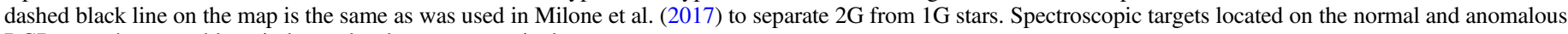
RGBs are shown as blue circles and red stars, respectively.
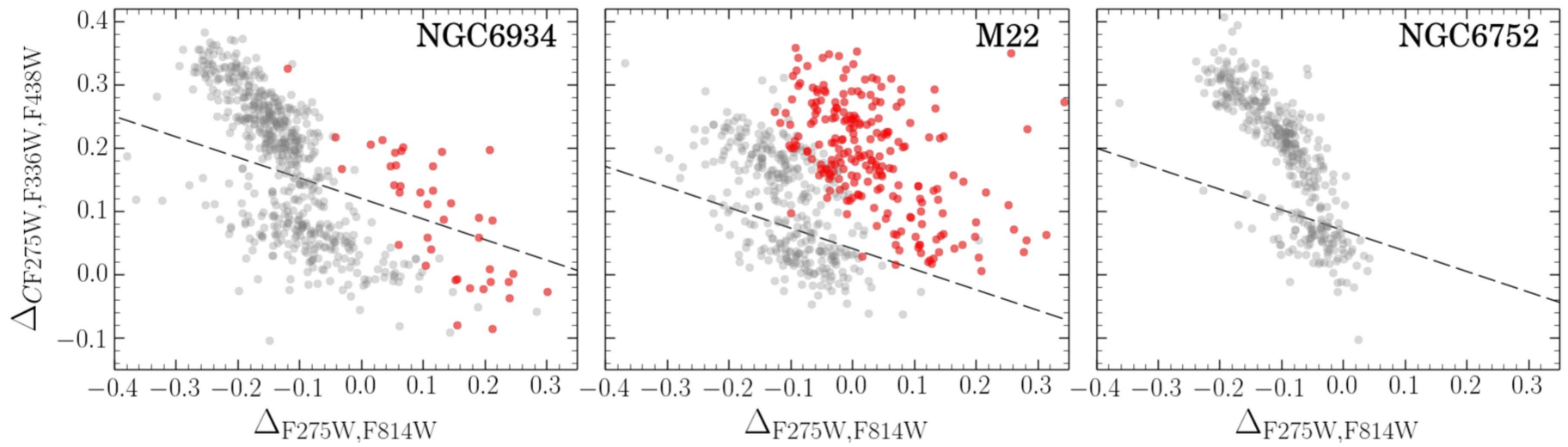

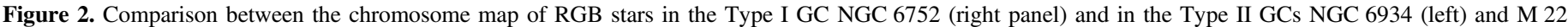

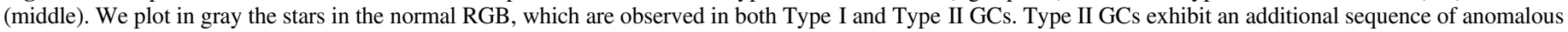
RGB stars, which are plotted in red. In all the panels, the dashed black line separates $2 \mathrm{G}$ from $1 \mathrm{G}$ stars, as in Milone et al. (2017).

metallicity and in heavier elements, specifically in elements produced via slow neutron-capture reactions ([s-elements $/ \mathrm{Fe}]$ ). Type II GCs include NGC 1851 (Yong \& Grundahl 2008), M 22 (Marino et al. 2009, Marino et al. 2011a; Lee 2016), NGC 5286 (Marino et al. 2015), M 2 (Yong et al. 2014), and, the most extreme case, $\omega$ Centauri (e.g., Norris \& Da Costa 1995; Johnson \& Pilachowski 2010; Marino et al. 2011a). In all cases variations in $[s$-elements $/ \mathrm{Fe}]$ are positively correlated with Fe. Furthermore, in NGC 1851 (Yong et al. 2009, 2015), M 22 (Marino et al. 2011a), and $\omega$ Centauri (Marino et al. 2012a) a difference in the overall $\mathrm{C}+\mathrm{N}+\mathrm{O}$ abundance has been found, with the stars enriched in $[s$-elements $/ \mathrm{Fe}]$ also having higher $[\mathrm{C}+\mathrm{N}+\mathrm{O} / \mathrm{Fe}]$ abundances. As stellar populations with different $\mathrm{C}+\mathrm{N}+\mathrm{O}$ are expected to have distinct luminosities on the sub-giant branch (SGB), these chemical variations are considered to play an important role in generating the split SGBs observed in these three GCs (Cassisi et al. 2008; Milone et al. 2008; Marino et al. 2012a, 2012b).

The complex chemical pattern of anomalous clusters and its resemblance on a less extreme scale to $\omega$ Centauri have raised the intriguing idea that these GCs could have originated in extra-Galactic environments (Bekki \& Freeman 2003). Specifically, these GCs could be the surviving nuclei of nowdisrupted dwarf galaxies, similar to what has been proposed to explain the chemical variations in $\omega$ Centauri. Such a dwarf galaxy environment could explain the capability of these GCs to support more extended star formation histories than typical GCs, which show variations in light elements only.

In this context, M54 stands out as an intriguing case (Carretta et al. 2010). It is the nuclear star cluster of the Sagittarius dwarf galaxy (Bellazzini et al. 2008) and certainly formed and evolved in an extra-Galactic environment. The photometric and spectroscopic similarities between M 54 and the other anomalous GCs makes it tempting to speculate that the latter are indeed remnants of dwarf galaxies cannibalized by the Milky Way.

To date, heavy-element variations have been observed in 11 Galactic GCs. Most surprisingly, the HST UV Survey has revealed that a relatively large fraction of the total sample of the 57 observed clusters, $\sim 18 \%$, belongs to the class of Type II GCs. We do not know yet if all Type II GCs can also be classified as anomalous from their chemistry, but all the anomalous GCs behave as Type II, based on their chromosome maps. The idea that such a large fraction of GCs $(\sim 18 \%)$ could be chemically anomalous would mean that many GCs in the Milky Way had a deeper potential well with which to retain high-velocity supernovae ejecta required to explain metallicity variations, and possibly be dwarf galaxy remnants. 
Table 1

Observation Details, Coordinates, and Radial Velocities for our Spectroscopic Targets

\begin{tabular}{|c|c|c|c|c|c|c|c|c|}
\hline ID & Spectrum & $\begin{array}{l}\text { Exp Time } \\
{[\mathrm{s}]}\end{array}$ & Obs-date & Airmass & $\begin{array}{l}\text { R.A. } \\
\text { J2000 }\end{array}$ & $\begin{array}{l}\text { Decl. } \\
\text { J2000 }\end{array}$ & $\begin{array}{c}\mathrm{RV} \\
\left(\mathrm{km} \mathrm{s}^{-1}\right)\end{array}$ & $\begin{array}{l}\mathrm{RV}_{\text {helio }} \\
\left(\mathrm{km} \mathrm{s}^{-1}\right)\end{array}$ \\
\hline \multirow[t]{2}{*}{ NGC6934-n2 } & MIKE & $2 \times 1800$ & 2017 May 05 & $\sim 1.5$ & $20: 34: 10.694$ & $07: 24: 19.393$ & -433.37 & -406.29 \\
\hline & GRACES & $4 \times 1050$ & 2015 Aug 13 & $\sim 1.1$ & & & -404.15 & -407.93 \\
\hline \multirow[t]{2}{*}{ NGC6934-a2 } & MIKE & $2 \times 1800$ & 2017 May 05 & $\sim 1.3$ & 20:34:07.579 & $07: 24: 17.220$ & -427.70 & -400.59 \\
\hline & GRACES & $2 \times 1000-2 \times 1020$ & 2015 Dec $15-16$ & $\sim 1.9$ & & & -380.23 & -401.37 \\
\hline NGC6934-n1 & GRACES & $4 \times 1010$ & 2015 Aug 13 & $\sim 1.3$ & $20: 34: 10.399$ & $07: 24: 17.964$ & -407.95 & -411.89 \\
\hline NGC6934-a1 & GRACES & $2 \times 1550$ & 2015 Dec $18-22$ & $\sim 1.9$ & $20: 34: 16.460$ & $07: 24: 53.374$ & -382.42 & -402.09 \\
\hline
\end{tabular}

In the present study we continue our investigation of Type II GC properties. For that purpose, we focus on the chemical abundances of a poorly studied GC, NGC 6934, which exhibits an unusual chromosome map. This metal-intermediate cluster $([\mathrm{Fe} / \mathrm{H}]=-1.47$, Harris 1996 , as updated in 2010) has been classified as a Type II GC since it hosts a split SGB visible in the optical filters that is connected with a red red giant branch (RGB) that is absent in typical Milky Way GCs (Milone et al. 2017). On the other hand, the position of these red RGB stars is slightly different from what is observed in anomalous GCs, as they lie at lower average $\Delta_{C}$ F275W,F336W,F438W values. Proper motions suggest that the red sequence stars on the chromosome map are cluster members, but they have never been investigated for chemical abundances. To better understand the chemical properties of multiple stellar populations in NGC 6934, we have selected four targets, two in each of the two sequences identified in its chromosome map.

The outline of the paper is as follows: Section 2 is a description of the spectroscopic and photometric data sets; the choice of the adopted atmospheric parameters is discussed in Section 3, and our chemical abundance analysis is outlined in Section 4. Section 5 describes the results, which are summarized and discussed in Section 6.

\section{Data \\ 2.1. The Photometric Data set: the Chromosome Map of NGC 6934}

The photometric data used in this study come from the HST UV-Legacy Survey, which investigated multiple stellar populations in GCs (GO-13297, Piotto et al. 2015). Details on the images analyzed and on the data reduction can be found in Piotto et al. (2015) and Milone et al. (2017). Photometry has been corrected for differential reddening effects, which are very small, $\Delta(E(B-\mathrm{V})) \lesssim 0.004 \mathrm{mag}$, for NGC 6934.

Milone et al. (2017) analyzed the chromosome map of 57 GCs, including NGC 6934, and noted some peculiarities (see their Figure 4) for this cluster with respect to typical GC maps. The chromosome map of NGC 6934 red giants is reproduced in the left panel of Figure 1, with the dashed line separating $2 \mathrm{G}$ from 1G stars as defined in Milone et al. (2017). In this parameter space we can clearly distinguish (i) $1 \mathrm{G}$ and $2 \mathrm{G}$ stars as the stars located below and above the dashed line, respectively; and (ii) two distinct patterns of stars represented with gray and red dots. The separate distribution of red stars on the chromosome map is a distinctive feature of the Type II GCs.

Another distinctive feature of Type II GCs is the presence of a split SGB in both optical and UV color-magnitude diagrams (CMDs). As shown in the right panel of Figure 1, the faint SGB of NGC 6934 is clearly connected with the red RGB in the $m_{\mathrm{F} 336 \mathrm{~W}}$ versus $m_{\mathrm{F} 336 \mathrm{~W}}-m_{\mathrm{F} 814 \mathrm{~W}} \mathrm{CMD}$. Red stars on the chromosome map have been selected as the stars defining the red $\mathrm{RGB}$ on the $\mathrm{CMD}$, represented in the right panel of Figure 1.

From its optical and ultraviolet CMDs and from its chromosome map, NGC 6934 has been classified as a Type II GC, but as discussed in Section 1, the comparison of the chromosome map of NGC 6934 with that observed in anomalous GCs suggests that the majority of red stars in anomalous GCs have higher values on the $\Delta_{C}$ F275W,F336W,F438W axis. This difference in the distribution of the stars can be easily seen in Figure 2, where we represent the chromosome map of NGC 6934 (left panel) in comparison with that of the anomalous GC M 22 (middle panel). For comparison purposes, we also display the chromosome map of the Type I GC NGC 6752 (right panel), which exhibits the presence of just $1 \mathrm{G}$ and $2 \mathrm{G}$ stars. In all the three GCs shown in Figure 2, the dashed line represents the separation between $1 \mathrm{G}$ and $2 \mathrm{G}$ stars, as defined in Milone et al. (2017). It is clear that while in M 22 all the red stars have $\Delta_{C}$ F275W,F336W,F438W values above the $1 \mathrm{G}-2 \mathrm{G}$ separation line, some red stars in NGC 6934 lie below this line.

To avoid confusion, we repeat here the terminology that is used throughout the paper. First, according to their chemical compositions, GCs are classified into normal and anomalous (e.g., Marino et al. 2015). Following this classification, in this paper the terms normal and anomalous refer to the chemical pattern observed in GCs from spectroscopy:

1. normal are the monometallic systems with the typical chemical patterns observed in GCs, e.g., the (anti-) correlations in light elements; and

2. anomalous will indicate GCs with unusual chemical pattern in heavy elements, e.g., variations in the $[s$-element $/ \mathrm{Fe}]$ ratios and/or $[\mathrm{Fe} / \mathrm{H}]$.

Second, according to their photometric pattern (Milone et al. 2017), GCs are identified as

1. Type I, which is used for GCs with single SGBs in CMDs from photometry with optical filters. These objects display the typical chromosome map characterized by a single sequence.

2. Type II, which designates those GCs with a split SGB in optical filter CMDs. These clusters exhibit a blue and red $\mathrm{RGB}$ in the $m_{\mathrm{F} 336 \mathrm{~W}}$ versus $m_{\mathrm{F} 336 \mathrm{~W}}-m_{\mathrm{F} 814 \mathrm{~W}} \mathrm{CMD}$. Stars on the red RGB also define additional red sequences on the chromosome map.

The synergy between photometry and spectroscopy reveals that (i) normal GCs exhibit the CMDs and the chromosome maps of Type I GCs; (ii) all the anomalous GCs analyzed so far are Type II, and the stars with enhanced Fe and/or [s-element/Fe] populate the red RGB. In the following we also refer to individual stars in Type II GCs as 
Table 2

Atmospheric Parameters, Derived from Spectroscopy and Photometry, and Corresponding Fe I and Fe II Abundances (with the Associated $\sigma$ and Number of Spectral Lines) for Our Targets

ID

SOURCE

$\begin{array}{lll}T_{\text {eff }} & \log g & {[\mathrm{~A} / \mathrm{H}]} \\ (\mathrm{K}) & (\mathrm{cgs}) & (\mathrm{dex})\end{array}$

$\begin{array}{cccc}T_{\text {eff }} & \log g & {[\mathrm{~A} / \mathrm{H}]} & \xi_{\mathrm{t}} \\ (\mathrm{K}) & (\mathrm{cgs}) & (\mathrm{dex}) & \left(\mathrm{km} \mathrm{s}^{-1}\right)\end{array}$

$\log \epsilon(\mathrm{Fe} \mathrm{I})$

$\sigma \quad \# \quad \log \epsilon$ (Fe II)

Spectroscopic

$\sigma$

$\begin{array}{cccc}T_{\text {eff }} & \log g & {[\mathrm{~A} / \mathrm{H}]} & \xi_{\mathrm{t}} \\ (\mathrm{K}) & (\mathrm{cgs}) & (\mathrm{dex}) & \left(\mathrm{km} \mathrm{s}^{-1}\right)\end{array}$

NGC6934-n2

$\begin{array}{lllll}\text { MIKE } & 4450 & 1.25 & -1.53 & 1.78\end{array}$

$\begin{array}{lllll}\text { GRACES } & 4500 & 1.10 & -1.37 & 1.77\end{array}$

$5.968 \quad 0.122$

$0.122 \quad 153$

$\begin{array}{llll}5.968 & 0.122 & 153 & 6.058 \\ 6.127 & 0.142 & 119 & 6.205 \\ 6.143 & 0.128 & 157 & 6.240\end{array}$

6.205
6.240

$\begin{array}{llllll}0.097 & 17 & 4372 & 1.22 & -1.61 & 1.78 \\ 0.169 & 10 & 4372 & 1.22 & -1.46 & 1.69\end{array}$

NGC6934-a2

NGC6934-n1

$\begin{array}{lllll}\text { MIKE } & 4450 & 1.40 & -1.35 & 1.80\end{array}$

$\begin{array}{llll}6.361 & 0.196 & 106 & 6.425\end{array}$

5.886
6.038

$\begin{array}{lllllll}0.169 & 10 & 4372 & 1.22 & -1.46 & 1.69 & 6.038 \\ 0.133 & 18 & 4380 & 1.30 & -1.39 & 1.75 & 6.096\end{array}$

\begin{tabular}{lll}
0.126 & 6.105 & 0.105 \\
0.149 & 6.398 & 0.172 \\
0.128 & 6.282 & 0.141 \\
0.204 & 6.494 & 0.114 \\
0.146 & 6.287 & 0.114 \\
0.178 & 6.648 & 0.230 \\
\hline
\end{tabular}

Note. Results are listed for both MIKE and GRACES spectra.

$\begin{array}{llll}6.361 & 0.196 & 106 & 6.425 \\ 6.108 & 0.141 & 112 & 6.188\end{array}$

$\begin{array}{rrrrrrr}0.113 & 8 & 4380 & 1.30 & -1.27 & 1.90 & 6.23\end{array}$

$\begin{array}{rrrrrrr}0.113 & 8 & 4380 & 1.30 & -1.27 & 1.90 & 6.23 \\ 0.101 & 11 & 4329 & 1.13 & -1.48 & 1.88 & 6.01 \\ 0.262 & 4 & 4459 & 1.48 & -1.25 & 1.85 & 6.25\end{array}$


Table 3

Line List with the Measured EWs for the Program Stars Observed with MIKE and GRACES

\begin{tabular}{|c|c|c|c|c|c|c|c|c|c|}
\hline $\begin{array}{l}\text { Wavelength } \\
{\left[\AA \AA^{\circ}\right]}\end{array}$ & Species & $\begin{array}{l}\text { L.E.P. } \\
{[\mathrm{eV}]}\end{array}$ & $\log g f$ & \multicolumn{2}{|c|}{$\mathrm{EWS}_{\mathrm{MIKE}}(\mathrm{m} \AA)$} & \multicolumn{4}{|c|}{$\mathrm{EWs}_{\mathrm{GRACES}}(\mathrm{m} \AA)$} \\
\hline 4139.94 & Fe I & 0.99 & -3.63 & 85.7 & 107.2 & $\cdots$ & $\ldots$ & $\cdots$ & $\cdots$ \\
\hline 4439.89 & $\mathrm{Fe} \mathrm{I}$ & 2.28 & -3.00 & 69.7 & 70.7 & $\ldots$ & $\ldots$ & $\ldots$ & $\ldots$ \\
\hline 4445.48 & $\mathrm{Fe} \mathrm{I}$ & 0.09 & -5.44 & 92.3 & 99.6 & $\ldots$ & $\ldots$ & $\ldots$ & $\ldots$ \\
\hline 4489.75 & $\mathrm{Fe} \mathrm{I}$ & 0.12 & -3.97 & 152.4 & 156.4 & $\ldots$ & $\ldots$ & $\ldots$ & $\ldots$ \\
\hline 4574.23 & $\mathrm{Fe} \mathrm{I}$ & 3.21 & -2.36 & 34.9 & 37.9 & $\ldots$ & $\ldots$ & $\ldots$ & $\ldots$ \\
\hline 4602.01 & $\mathrm{Fe} \mathrm{I}$ & 1.61 & -3.15 & 100.2 & 112.0 & 90.6 & $\ldots$ & 133.9 & $\ldots$ \\
\hline 4602.95 & $\mathrm{Fe} \mathrm{I}$ & 1.49 & -2.22 & 148.0 & 164.4 & 148.7 & $\ldots$ & $\ldots$ & $\ldots$ \\
\hline 4658.30 & $\mathrm{Fe} \mathrm{I}$ & 3.27 & -2.96 & $\ldots$ & 18.8 & $\ldots$ & $\ldots$ & $\ldots$ & $\ldots$ \\
\hline 4788.76 & $\mathrm{Fe} \mathrm{I}$ & 3.24 & -1.54 & 61.9 & 71.9 & 71.7 & $\ldots$ & $\ldots$ & $\ldots$ \\
\hline 4839.55 & $\mathrm{Fe} \mathrm{I}$ & 3.27 & -1.84 & 61.7 & 73.6 & 70.2 & $\ldots$ & $\cdots$ & $\cdots$ \\
\hline 4859.74 & $\mathrm{Fe} \mathrm{I}$ & 2.87 & -0.78 & 139.0 & 149.6 & $\ldots$ & $\ldots$ & $\ldots$ & $\ldots$ \\
\hline 4885.43 & $\mathrm{Fe} \mathrm{I}$ & 3.88 & -1.15 & 58.6 & 61.0 & $\ldots$ & $\ldots$ & $\ldots$ & $\ldots$ \\
\hline 4917.24 & $\mathrm{Fe} \mathrm{I}$ & 4.19 & -1.27 & 45.1 & 52.3 & 59.1 & $\ldots$ & 41.7 & $\cdots$ \\
\hline 4924.77 & $\mathrm{Fe} \mathrm{I}$ & 2.28 & -2.29 & 111.2 & 122.8 & 114.8 & $\ldots$ & 128.9 & $\ldots$ \\
\hline
\end{tabular}

Note. Only a portion of this table is shown here to demonstrate its form and content.

(This table is available in its entirety in machine-readable form.)

1. blue or normal RGB stars if they define the blue RGB and fall in the typical chromosome map sequence, as observed in all GCs;

2. red or anomalous RGB stars if they are located on the additional red sequences, observed in Type II GCs only; and

3. in this paper, the targets on the normal and anomalous RGBs are designated $\mathrm{n} 1$ and $\mathrm{n} 2$, and $\mathrm{a} 1$, and a2, respectively.

\subsection{The Photometric Data Set: The Chromosome Map of NGC 6934}

Our spectroscopic data have been acquired using the Gemini Remote Access to Canada-France-Hawaii Telescope (CFHT) ESPaDOnS (Donati 2003) Spectrograph (GRACES; Chené et al. 2014) through the program GN-2015B-Q-81, and with the Magellan Inamori Kyocera Echelle (MIKE, Bernstein et al. 2003) spectrograph on the Magellan-clay $6.5 \mathrm{~m}$ telescope. The targets were four RGB stars with $V \sim 15$ mag selected from the chromosome map described in Section 2.1. Their locations in the chromosome map and in the $m_{\mathrm{F} 336 \mathrm{~W}^{-}}\left(m_{\mathrm{F} 336 \mathrm{~W}}-m_{\mathrm{F} 814 \mathrm{~W}}\right)$ CMD of NGC 6934 are shown in Figure 1. We have chosen two giants lying on the normal RGB and two on the anomalous RGB, represented with blue filled circles and red stars, respectively. As our primary goal is to study the heavy-element pattern, we note here that our spectroscopic samples are biased toward relatively low values of $\Delta_{C}$ F275W,F336W,F438W. This choice was made to avoid contamination from stars characterized by substantially different abundances of nitrogen and/or other light elements (Milone et al. 2017). Furthermore, our selected targets are among the brightest $1 \mathrm{G}$ stars in order to maximize the signal-to-noise ratio $(\mathrm{S} / \mathrm{N})$ of the spectra. The observing log is given in Table 1.

High-resolution $(R \sim 40,000)$ optical spectra were obtained with GRACES in August and December 2015 using the target + sky two-fiber spectroscopic mode. Briefly, light from the Gemini North telescope is fed to the Echelle SpectroPolarimetric Device for the Observation of Stars (ESPaDOnS) at the CFHT via two $270 \mathrm{~m}$ long optical fibers that have $\sim 8 \%$ throughput (see Chené et al. 2014). Basic data reduction was performed using the OPERA pipeline (Martioli et al. 2012,
Malo et al., in preparation). Two of the four targets observed with GRACES were subsequently reobserved with MIKE with the aim of gathering an independent analysis from a different data set. Specifically, we gathered MIKE spectra for one target on the normal sequence, and one on the anomalous sequence, chosen to have very similar effective temperatures based on the analysis of the GRACES data. The MIKE spectra were taken in 2017 May, using a 0 " 70 slit, which gives $R \sim 35,000$ in the blue and $R \sim 30,000$ in the red, respectively. Data reduction involving bias-subtraction, flat-field correction, wavelengthcalibration, and sky-subtraction has been done using the dedicated CarPy pipeline ${ }^{5}$ (Kelson et al. 2000; Kelson 2003).

Both for the GRACES and MIKE spectra, coaddition of the individual exposures, continuum normalization, radial velocity (RV) determination, and correction to laboratory wavelength was performed using IRAF routines. The spectral coverage for the GRACES spectra is $\sim 4070-10020 \AA$, while the MIKE spectra cover the spectral region from $\sim 3350 \AA$ to $\sim 9420 \AA$. The $\mathrm{S} / \mathrm{N}$ of our MIKE spectra is $\sim 120$ at $6000 \AA$, while the GRACES spectra have a lower $\mathrm{S} / \mathrm{N}$, between 40 and 70 at $6000 \AA$ A. The chemical abundance analysis was performed by neglecting the bluest part of the spectra because of the low $\mathrm{S} / \mathrm{N}$. Specifically, we have analyzed the spectral regions with $\lambda \gtrsim 4200 \AA$ for MIKE and $\lambda \gtrsim 4500 \AA$ for GRACES.

Radial velocities were derived using the IRAF@FXCOR task, which cross-correlates the object spectrum with a template. For the template we used a synthetic spectrum generated with the latest version of $\mathrm{MOOG}^{6}$ (Sneden 1973). This spectrum was computed with a model stellar atmosphere interpolated from the Castelli \& Kurucz (2004) grid, ${ }^{7}$ adopting parameters (effective temperature $\left(T_{\text {eff }}\right)$, surface gravity $(\log g)$, microturbulent velocity $\left(\xi_{\mathrm{t}}\right)$, and overall metallicity $\left.([\mathrm{A} / \mathrm{H}])\right)$ of $4600 \mathrm{~K}, 2.5,2.0 \mathrm{~km} \mathrm{~s}^{-1}$, and -1.45 dex, respectively. Observed $\mathrm{RVs}$ were then corrected to the heliocentric system.

Heliocentric RVs were used as a proof of cluster membership for our targets. We measure an average RV for the four stars of

\footnotetext{
5 See http://code.obs.carnegiescience.edu/mike.

6 http://www.as.utexas.edu/ chris/moog.html

7 http://kurucz.harvard.edu/grids.html
} 
Table 4

Analyzed Chemical Abundances from $\mathrm{C}$ to $\mathrm{Ni}$

\begin{tabular}{|c|c|c|c|c|c|c|c|c|c|c|c|c|c|c|c|c|c|c|}
\hline ID & Spectrum & {$[\mathrm{C} / \mathrm{Fe}]$} & $\sigma$ & $\#$ & {$[\mathrm{O} / \mathrm{Fe}]$} & $\sigma$ & $\#$ & {$[\mathrm{Na} / \mathrm{Fe}]$} & $\sigma$ & {$[\mathrm{Na} / \mathrm{Fe}]_{\mathrm{NLTE}}$} & $\sigma$ & $\#$ & {$[\mathrm{Mg} / \mathrm{Fe}]$} & $\sigma$ & $\#$ & {$[\mathrm{Al} / \mathrm{Fe}]$} & $\sigma$ & $\overline{\#}$ \\
\hline \multirow[t]{2}{*}{ NGC6934-n2 } & MIKE & -0.58 & 0.02 & 2 & 0.57 & 0.01 & 2 & 0.05 & 0.24 & -0.13 & 0.08 & 5 & 0.39 & 0.00 & 2 & -0.20 & 0.13 & $\overline{2}$ \\
\hline & GRACES & $\ldots$ & $\ldots$ & $\ldots$ & 0.50 & 0.08 & 2 & 0.08 & 0.31 & -0.08 & 0.30 & 3 & 0.37 & 0.04 & 2 & -0.14 & 0.17 & 2 \\
\hline \multirow[t]{2}{*}{ NGC6934-a2 } & MIKE & -0.56 & 0.01 & 2 & 0.72 & 0.08 & 2 & 0.00 & 0.23 & -0.17 & 0.07 & 6 & 0.36 & 0.00 & 2 & -0.15 & 0.05 & 2 \\
\hline & GRACES & $\ldots$ & $\ldots$ & $\ldots$ & 0.58 & 0.13 & 2 & -0.27 & 0.28 & -0.39 & 0.15 & 4 & 0.34 & 0.07 & 2 & $\ldots$ & $\ldots$ & $\ldots$ \\
\hline NGC6934-n1 & GRACES & $\ldots$ & $\cdots$ & $\cdots$ & 0.64 & 0.04 & 2 & 0.22 & 0.08 & 0.09 & 0.09 & 5 & 0.48 & 0.11 & 2 & +0.13 & 0.02 & 2 \\
\hline NGC6934-a1 & GRACES & $\cdots$ & $\cdots$ & $\cdots$ & 0.63 & 0.13 & 2 & 0.17 & $\cdots$ & -0.18 & $\cdots$ & 1 & 0.54 & $\cdots$ & 1 & -0.08 & $\cdots$ & 1 \\
\hline ID & Spectrum & {$[\mathrm{Si} / \mathrm{Fe}]$} & & & \# & {$[\mathrm{Ca} / \mathrm{Fe}]$} & $\sigma$ & $\#$ & {$[\mathrm{Sc} / \mathrm{Fe}] \mathrm{II}$} & $\sigma$ & \# & {$[\mathrm{Ti} / \mathrm{Fe}] \mathrm{I}$} & $\sigma$ & \# & & /Fe] II & $\sigma$ & \# \\
\hline \multirow[t]{2}{*}{ NGC6934-n2 } & MIKE & 0.38 & & & 9 & 0.32 & 0.10 & 17 & 0.05 & 0.20 & 6 & 0.19 & 0.10 & 29 & & .35 & 0.16 & $\overline{9}$ \\
\hline & GRACES & 0.55 & & & 4 & 0.33 & 0.09 & 17 & 0.00 & 0.27 & 7 & 0.14 & 0.23 & 21 & & .32 & 0.34 & 3 \\
\hline \multirow[t]{2}{*}{ NGC6934-a2 } & MIKE & 0.35 & & & 11 & 0.32 & 0.10 & 19 & 0.09 & 0.12 & 7 & 0.17 & 0.16 & 32 & & .33 & 0.12 & 10 \\
\hline & GRACES & 0.47 & & & 2 & 0.35 & 0.24 & 18 & -0.13 & 0.35 & 4 & 0.21 & 0.16 & 16 & & .12 & 0.18 & 2 \\
\hline NGC6934-n1 & GRACES & 0.50 & & & 4 & 0.25 & 0.12 & 16 & -0.06 & 0.12 & 6 & 0.21 & 0.25 & 18 & & .32 & 0.15 & 4 \\
\hline NGC6934-a1 & GRACES & 0.53 & & & 1 & 0.33 & 0.20 & 12 & $\ldots$ & $\cdots$ & $\cdots$ & 0.15 & 0.09 & 8 & & $\cdots$ & $\cdots$ & $\cdots$ \\
\hline ID & Spectrum & {$[\mathrm{V} / \mathrm{Fe}]$} & $\sigma$ & \# & {$[\mathrm{Cr} / \mathrm{Fe}] \mathrm{I}$} & $\sigma$ & $\#$ & {$[\mathrm{Cr} / \mathrm{Fe}] \mathrm{II}$} & $\sigma$ & {$[\mathrm{Mn} / \mathrm{Fe}]$} & $\sigma$ & \# & {$[\mathrm{Co} / \mathrm{Fe}]$} & $\sigma$ & \# & {$[\mathrm{Ni} / \mathrm{Fe}]$} & $\sigma$ & \# \\
\hline \multirow[t]{2}{*}{ NGC6934-n2 } & MIKE & 0.03 & 0.11 & 14 & -0.11 & 0.09 & 10 & 0.14 & $\ldots$ & -0.46 & 0.13 & 9 & -0.13 & $\ldots$ & 1 & -0.05 & 0.14 & 24 \\
\hline & GRACES & -0.07 & 0.14 & 10 & -0.03 & 0.17 & 8 & -0.06 & $\ldots$ & -0.62 & 0.15 & 9 & 0.02 & $\ldots$ & 1 & +0.07 & 0.24 & 14 \\
\hline \multirow[t]{2}{*}{ NGC6934-a2 } & MIKE & 0.05 & 0.09 & 14 & -0.11 & 0.09 & 10 & 0.16 & 0.24 & -0.42 & 0.08 & 9 & -0.19 & 0.09 & 2 & -0.01 & 0.14 & 23 \\
\hline & GRACES & 0.02 & 0.18 & 10 & -0.09 & 0.38 & 5 & $\ldots$ & $\ldots$ & -0.66 & 0.09 & 5 & $\ldots$ & $\ldots$ & $\ldots$ & -0.09 & 0.14 & 14 \\
\hline NGC6934-n1 & GRACES & -0.09 & 0.12 & 9 & -0.06 & 0.30 & 4 & $\ldots$ & $\ldots$ & -0.62 & 0.12 & 5 & -0.06 & $\ldots$ & 1 & +0.01 & 0.13 & 17 \\
\hline NGC6934-a1 & GRACES & 0.10 & 0.14 & 6 & $\ldots$ & $\ldots$ & $\ldots$ & $\ldots$ & $\ldots$ & -0.59 & 0.14 & 5 & -0.23 & $\ldots$ & 1 & -0.01 & 0.16 & 7 \\
\hline
\end{tabular}


$\langle\mathrm{RV}\rangle=-405.5 \pm 2.9 \mathrm{~km} \mathrm{~s}^{-1}\left(\mathrm{rms}=5.0 \mathrm{~km} \mathrm{~s}^{-1}\right)$, which is consistent with the Harris tabulated value of $-411.4 \pm$ $1.6 \mathrm{~km} \mathrm{~s}^{-1}$ (Harris 2010). Such extreme negative RVs are clearly distinct from the RVs of typical field stars. Hence, based on both RVs and proper motions, we can be confident of the membership for all four targets. Coordinates and RVs, both observed (RV) and corrected to the heliocentric system $\left(\mathrm{RV}_{\text {helio }}\right)$, for all four stars are listed in Table 1.

\section{Model Atmospheres}

The relatively high resolution and the large spectral coverage of our spectra allowed us to have a fully spectroscopic estimate of the stellar parameters, $T_{\mathrm{eff}}, \log g,[\mathrm{~A} / \mathrm{H}]$, and $\xi_{\mathrm{t}}$. Hence, we determined $T_{\text {eff }}$ by imposing the excitation potential equilibrium of the FeI lines and gravity with the ionization equilibrium between Fe I and Fe II lines. Note that for $\log g$ we impose Fe II abundances that are 0.05-0.07 dex higher than those of $\mathrm{Fe}$ I to adjust for non-local thermodynamic equilibrium (non-LTE) effects (Bergemann et al. 2012; Lind et al. 2012). For this analysis, $\xi_{\mathrm{t}}$ was set to minimize any dependence of Fe I abundances as a function of equivalent width $(\mathrm{EW})$. For the two stars observed with both GRACES and MIKE, we derived the atmospheric parameters separately for each spectrum.

As an independent test of our results, we also derived atmospheric parameters from our HST photometry (see Section 2.1). The $m_{\mathrm{F} 606 \mathrm{~W}}$ and $m_{\mathrm{F} 814 \mathrm{~W}}$ mag have been converted into $V$ and $I$ mag (Anderson et al. 2008), which we then used to estimate temperatures from the Alonso et al. (1999) colortemperature calibrations. The use of the $(V-\mathrm{I})$ color for this purpose is justified by the fact that it is mostly insensitive to variations in light elements. Surface gravities were then obtained from the apparent $V$ magnitudes, the photometric $T_{\text {eff }}$, bolometric corrections from Alonso et al. (1999), apparent distance modulus of $(m-M)_{V}=16.28$ (Harris 2010), and masses of $0.80 M_{\odot}$. Once $T_{\text {eff }}$ and $\log g$ have been fixed from photometry, we derived $\xi_{\mathrm{t}}$ from the $\mathrm{Fe}$ I lines as explained above. The atmospheric parameters obtained from spectroscopy and photometry are listed in Table 2.

By comparing the spectroscopic stellar parameters obtained from the GRACES and MIKE spectra for the two stars observed with both instruments, we note that (i) GRACES $T_{\text {eff }}$ are only marginally higher, (ii) $\log g$ values agree within $\lesssim 0.20$ dex, and (iii) GRACES metallicities are systematically higher by $\sim 0.20$ dex. By adopting exactly the same atmospheric parameters from photometry (right columns in Table 2) for the MIKE and GRACES spectra, we have a mean difference in $[\mathrm{Fe} / \mathrm{H}]$ of 0.135 dex between stars observed with both the instruments, with higher values for GRACES. We have verified that this difference is introduced by the systematically higher EWs, by $\sim 6 \mathrm{m \AA}$, obtained from the GRACES spectra. We note here that the MIKE Fe abundances are in better accord with the tabulated Harris value $([\mathrm{Fe} / \mathrm{H}]=-1.47 \mathrm{dex}$; Harris 2010). As abundance offsets are not unexpected when using spectra from different spectrographs, in the following we consider results from different instruments separately, and average them appropriately only when necessary.

We adopt as our primary set of atmospheric parameters the values obtained from the MIKE spectra, as they have a higher $\mathrm{S} / \mathrm{N}$ and a larger number of measured Fe lines. For GRACES, due to the low number of measurable lines, in particular Fe II, we prefer to adopt the parameters based on photometry as our primary set. In the following, when appropriate, we use both the sets of parameters to check our results.

In order to have an estimate of the internal errors associated with our atmospheric parameters, we have compared our $T_{\text {eff }} / \log g$ values from the spectral lines with those derived from the $\left(V-\right.$ I) $H S T$ colors. We obtain $\Delta T_{\text {eff }}=T_{\text {eff Fe lines }}-T_{\text {eff }}$ $(V-\mathrm{I})=+126 \pm 21 \mathrm{~K}(\mathrm{rms}=48 \mathrm{~K})$, and $\Delta \log g=\log g_{\mathrm{Fe}}$ lines $\log g_{(V-I)}=+0.06 \pm 0.06 \quad(\mathrm{rms}=0.14)$. This comparison suggests that the spectroscopic $T_{\text {eff }} / \log g$ scales are systematically higher, but the internal errors in these parameters are smaller, comparable with the rms of the average differences, e.g., about $50 \mathrm{~K}$ and $0.15 \mathrm{dex}$, in temperature and gravity, respectively. Furthermore, in our chemical abundance analysis we adopt typical internal uncertainties of $0.20 \mathrm{~km} \mathrm{~s}^{-1}$ for $\xi_{\mathrm{t}}$ and 0.10 dex for metallicity.

\section{Chemical Abundance Analysis}

Chemical abundances were derived from an LTE analysis using the spectral analysis code MOOG (Sneden 1973) and the alpha-enhanced Kurucz model atmospheres of Castelli \& Kurucz (2004), whose parameters have been obtained as described in Section 3.

A list of our analyzed spectral lines, with their associated EWs, excitation potentials, and total oscillator strengths $(\log g f)$, is provided in Table 3 . The chemical abundances for all the elements, with the exception of those discussed below, have been inferred from an EW-based analysis. We now comment on some of the transitions that we used.

Proton-capture elements. We have derived abundance ratios with respect to $\mathrm{Fe}$ for $\mathrm{C}, \mathrm{O}, \mathrm{Na}, \mathrm{Al}$, and $\mathrm{Mg}$. Carbon has been inferred from spectral synthesis of the $\mathrm{CH} G$-band $\left(A^{2} \Delta-X^{2} \Pi\right)$ features near 4312 and $4323 \AA$. The $\mathrm{S} / \mathrm{N}$ of the GRACES spectra at these wavelengths was too low to derive abundances, so $\mathrm{C}$ has been measured only from MIKE spectra. Oxygen abundances were inferred from the synthesis of the forbidden [O I] lines at 6300 and $6363 \AA$. Although we have not derived any $3 \mathrm{D}$ non-LTE correction for these spectral lines, we note that from the grid by Amarsi et al. (2016) that they are small for metal-poor stars within their analyzed range of atmospheric parameters $\left(5000<T_{\text {eff }}<6500 \mathrm{~K}, 3.0<\right.$ $\log g<5.0 \mathrm{~g} / \mathrm{cm} \mathrm{s}^{-2}$ ). Our giants are, however, somewhat cooler, and have much lower surface gravities. Furthermore, telluric $\mathrm{O}_{2}$ and $\mathrm{H}_{2} \mathrm{O}$ spectral absorptions often affect the $\mathrm{O}$ line at $6300 \AA$. Indeed, for our targets, the analyzed $\mathrm{O}$ transition is contaminated by $\mathrm{O}_{2}$ lines. We have removed the telluric features by using the software MOLECFIT ${ }^{8}$ provided by ESO (Kausch et al. 2015; Smette et al. 2015). Nevertheless, even with such a subtraction procedure, we caution that residual telluric feature contamination might be a concern for the analysis of the 6300.3 [O I] line. We determined $\mathrm{Na}$ abundances from the EWs of the NaI doublets at $\sim 5680 \AA$, $\sim 6150 \AA$, and $\sim 8190 \AA$. Chemical abundances for this species have been corrected for non-LTE effects by following the recipes in Lind et al. (2011). Aluminum was inferred from the synthesis of the doublet at $\sim 6667 \AA$, while magnesium abundances were determined from the EWs of the transitions at $\sim 5528$ and $5711 \AA$.

Manganese. For Mn, we have synthetized the spectral lines at around 4710, 4739, 4754, 4762, 5395, 5420, 5433, 6014, and $6022 \AA$ by assuming $f\left({ }^{55} \mathrm{Mn}\right)=1.00$. When available, the

http://www.eso.org/sci/software/pipelines/skytools/molecfit 
Table 5

Analyzed Chemical Abundances from Cu to Dy

\begin{tabular}{|c|c|c|c|c|c|c|c|c|c|c|c|c|c|c|c|c|}
\hline ID & Spectrum & {$[\mathrm{Cu} / \mathrm{Fe}]$} & $\sigma$ & $\#$ & {$[\mathrm{Zn} / \mathrm{Fe}]$} & $\sigma$ & $\#$ & {$[\mathrm{Sr} / \mathrm{Fe}] \mathrm{II}$} & $\sigma$ & $\#$ & {$[\mathrm{Y} / \mathrm{Fe}] \mathrm{II}$} & $\sigma$ & $\#$ & {$[\mathrm{Zr} / \mathrm{Fe}] \mathrm{II}$} & $\sigma$ & \# \\
\hline \multirow[t]{2}{*}{ NGC6934-n2 } & MIKE & -0.59 & 0.16 & 2 & 0.15 & 0.01 & 2 & 0.55 & $\ldots$ & 1 & -0.01 & 0.02 & 4 & 0.24 & $\ldots$ & 1 \\
\hline & GRACES & -0.75 & 0.15 & 2 & $\cdots$ & $\cdots$ & $\cdots$ & $\cdots$ & $\cdots$ & $\cdots$ & -0.07 & 0.17 & 4 & $\cdots$ & $\cdots$ & $\cdots$ \\
\hline \multirow[t]{2}{*}{ NGC6934-a2 } & MIKE & -0.66 & 0.01 & 2 & 0.14 & 0.11 & 2 & 0.60 & $\ldots$ & 1 & 0.01 & 0.06 & 4 & 0.21 & $\cdots$ & 1 \\
\hline & GRACES & -0.73 & $\ldots$ & 1 & $\ldots$ & $\cdots$ & $\cdots$ & $\ldots$ & $\ldots$ & $\ldots$ & $\ldots$ & $\ldots$ & $\ldots$ & $\ldots$ & $\cdots$ & $\cdots$ \\
\hline NGC6934-n1 & GRACES & -0.80 & $\ldots$ & 1 & $\ldots$ & $\ldots$ & $\ldots$ & $\ldots$ & $\ldots$ & $\ldots$ & -0.06 & 0.10 & 2 & $\ldots$ & $\ldots$ & $\cdots$ \\
\hline NGC6934-a1 & GRACES & -0.75 & $\cdots$ & 1 & $\cdots$ & $\cdots$ & $\cdots$ & $\cdots$ & $\cdots$ & $\cdots$ & $\cdots$ & $\cdots$ & $\cdots$ & $\cdots$ & $\cdots$ & \\
\hline
\end{tabular}

\begin{tabular}{|c|c|c|c|c|c|c|c|c|c|c|c|c|c|}
\hline ID & Spectrum & {$[\mathrm{Ba} / \mathrm{Fe}] \mathrm{II}$} & $\sigma$ & $\#$ & {$[\mathrm{La} / \mathrm{Fe}] \mathrm{II}$} & $\sigma$ & $\#$ & {$[\mathrm{Ce} / \mathrm{Fe}] \mathrm{II}$} & $\sigma$ & $\#$ & {$[\mathrm{Pr} / \mathrm{Fe}] \mathrm{II}$} & $\sigma$ & \# \\
\hline \multirow[t]{2}{*}{ NGC6934-n2 } & MIKE & 0.09 & 0.09 & 3 & 0.26 & 0.09 & 8 & 0.22 & 0.07 & 7 & 0.31 & 0.03 & 2 \\
\hline & GRACES & 0.10 & 0.05 & 3 & 0.27 & 0.13 & 6 & 0.15 & $\ldots$ & 1 & 0.34 & $\ldots$ & 1 \\
\hline \multirow[t]{2}{*}{ NGC6934-a2 } & MIKE & 0.04 & 0.05 & 3 & 0.31 & 0.08 & 8 & 0.19 & 0.09 & 7 & 0.31 & 0.13 & 2 \\
\hline & GRACES & -0.01 & 0.06 & 3 & 0.20 & 0.05 & 2 & $\ldots$ & $\ldots$ & $\cdots$ & $\ldots$ & $\ldots$ & $\cdots$ \\
\hline NGC6934-n1 & GRACES & 0.10 & 0.08 & 3 & 0.23 & 0.12 & 6 & 0.19 & 0.03 & 2 & 0.21 & $\cdots$ & 1 \\
\hline NGC6934-a1 & GRACES & 0.17 & 0.23 & 3 & 0.10 & $\cdots$ & 1 & $\cdots$ & $\cdots$ & $\cdots$ & $\cdots$ & $\cdots$ & $\cdots$ \\
\hline ID & Spectrum & {$[\mathrm{Nd} / \mathrm{Fe}] \mathrm{II}$} & $\sigma$ & $\#$ & {$[\mathrm{Sm} / \mathrm{Fe}] \mathrm{II}$} & $\sigma$ & $\#$ & {$[\mathrm{Eu} / \mathrm{Fe}] \mathrm{II}$} & $\sigma$ & \# & {$[\mathrm{Dy} / \mathrm{Fe}] \mathrm{II}$} & $\sigma$ & $\#$ \\
\hline \multirow[t]{2}{*}{ NGC6934-n2 } & MIKE & 0.34 & 0.09 & 18 & 0.50 & 0.07 & 14 & 0.63 & 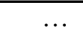 & 1 & 0.83 & $\cdots$ & $\overline{1}$ \\
\hline & GRACES & 0.36 & 0.09 & 10 & 0.59 & 0.17 & 3 & 0.50 & $\ldots$ & 1 & $\ldots$ & $\ldots$ & $\cdots$ \\
\hline \multirow[t]{2}{*}{ NGC6934-a2 } & MIKE & 0.33 & 0.14 & 18 & 0.50 & 0.13 & 14 & 0.53 & $\cdots$ & 1 & 0.82 & $\cdots$ & 1 \\
\hline & GRACES & 0.49 & 0.05 & 3 & $\cdots$ & $\cdots$ & $\cdots$ & 0.63 & $\cdots$ & 1 & $\cdots$ & $\cdots$ & $\cdots$ \\
\hline NGC6934-n1 & GRACES & 0.31 & 0.11 & 11 & 0.57 & 0.25 & 4 & 0.50 & $\cdots$ & 1 & $\cdots$ & $\cdots$ & \\
\hline NGC6934-a1 & GRACES & 0.36 & 0.04 & 3 & $\ldots$ & $\ldots$ & $\ldots$ & 0.55 & $\ldots$ & 1 & $\ldots$ & $\cdots$ & .. \\
\hline
\end{tabular}



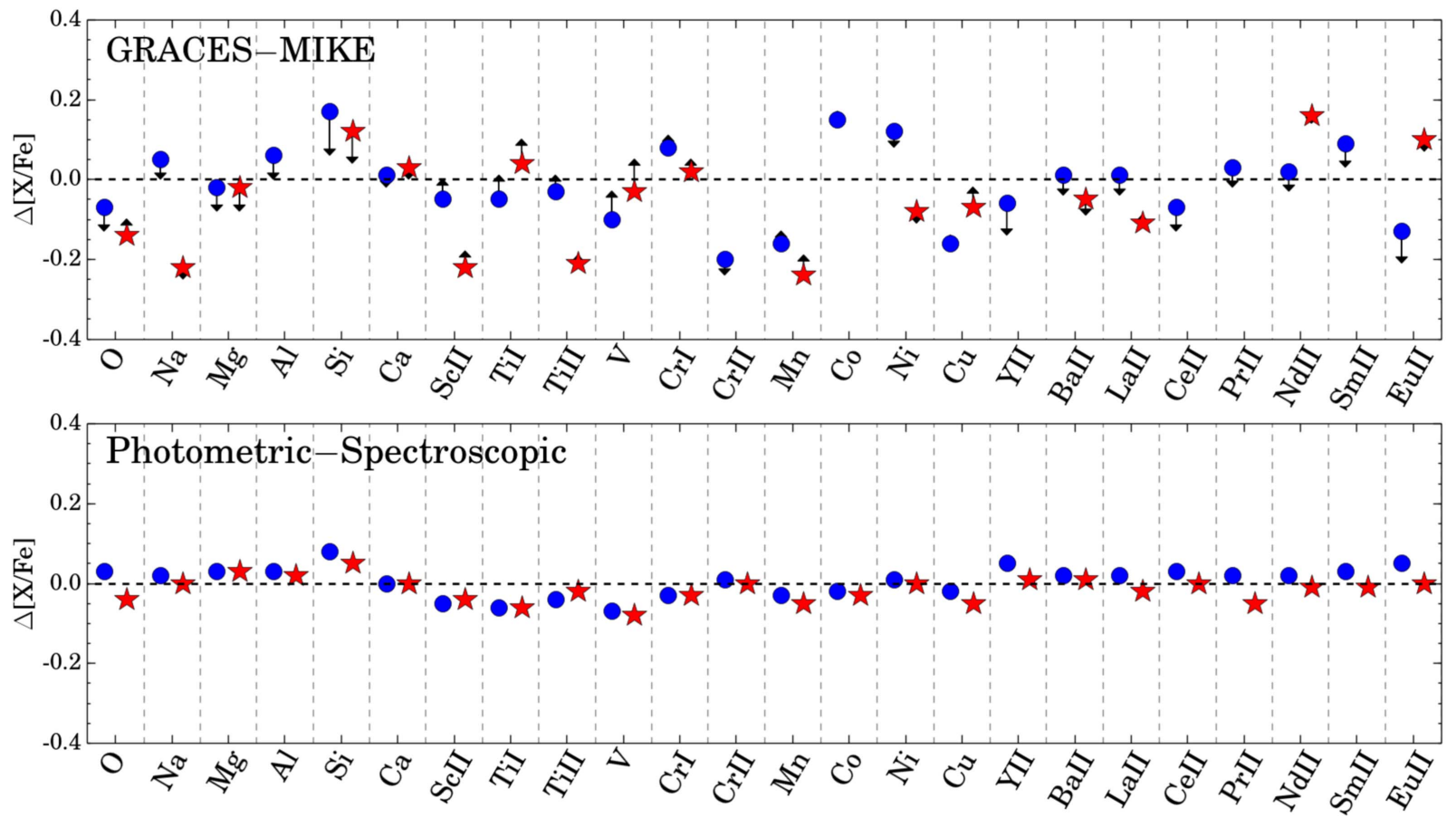

Figure 3. Upper panel: abundance ratio differences, $[\mathrm{X} / \mathrm{Fe}]_{\text {GRACES }}-[\mathrm{X} / \mathrm{Fe}]_{\text {MIKE }}$, obtained for the stars $\mathrm{n} 2$ (blue circle) and a2 (red star), observed with both the MIKE and GRACES spectrographs. Lower panel: abundance ratios differences, $[\mathrm{X} / \mathrm{Fe}]_{\text {phot }}-[\mathrm{X} / \mathrm{Fe}]_{\text {spectr }}$, derived for $\mathrm{n} 2$ and a2, observed with MIKE spectra, and analyzed by using stellar parameters from photometry $\left([\mathrm{X} / \mathrm{Fe}]_{\text {phot }}\right)$ and spectral Fe lines $\left([\mathrm{X} / \mathrm{Fe}]_{\text {spectr }}\right)$. The offsets $\left(\Delta[\mathrm{X} / \mathrm{Fe}]=[\mathrm{X} / \mathrm{Fe}]_{\text {phot }}-[\mathrm{X} / \mathrm{Fe}]_{\text {spectr }}\right)$ are shown in the upper panel with black arrows to visualize possible differences in the abundance ratios due to the different sets of atmospheric parameters assumed for MIKE and GRACES spectra.

hyperfine-splitting data have been taken from Lawler et al. (2001a, 2001b), otherwise from the Kurucz (2009) compendium. ${ }^{9}$

Copper. Abundances for $\mathrm{Cu}$ were inferred from synthesis of the $\mathrm{Cu}$ I lines at $5105,5782 \AA$. Both hyperfine and isotopic splitting were included in the analysis, using the well-studied spectral line component structure from Kurucz (2009). Solar system isotopic fractions were assumed in the computations: $f\left({ }^{63} \mathrm{Cu}\right)=0.69$ and $f\left({ }^{65} \mathrm{Cu}\right)=0.31$.

Neutron-capture elements. We derived $\mathrm{Sr}, \mathrm{Y}, \mathrm{Zr}, \mathrm{Ba}, \mathrm{La}, \mathrm{Ce}$, $\mathrm{Pr}, \mathrm{Nd}, \mathrm{Sm}, \mathrm{Eu}$, and Dy abundances from the MIKE spectra and $\mathrm{Y}, \mathrm{Ba}, \mathrm{La}, \mathrm{Ce}, \mathrm{Pr}, \mathrm{Nd}, \mathrm{Sm}$, and Eu from the GRACES spectra. For all these elements we performed a spectral synthesis analysis, as hyperfine and/or isotopic splitting and/ or blending features needed to be taken into account. In all the cases we have assumed solar system isotopic fractions.

The inferred chemical abundances are listed in Tables 4-5. Internal uncertainties in chemical abundances due to the adopted model atmospheres were estimated by varying the stellar parameters, one at a time, by the amounts derived in Section 3, namely $T_{\text {eff }} / \log g /[\mathrm{Fe} / \mathrm{H}] / \xi_{\mathrm{t}}= \pm 50 \mathrm{~K} / \pm 0.15 \mathrm{cgs} /$ $\pm 0.10 \mathrm{dex} / \pm 0.20 \mathrm{~km} \mathrm{~s}^{-1}$. Another way to evaluate how the adopted model atmospheres affect our results is by comparing chemical abundances for the same stars inferred from the spectroscopic and photometric parameters. This test is particularly enlightening since we have adopted spectroscopic parameters for MIKE data and photometric parameters for GRACES. In the lower panel of Figure 3 we plot the differences $\Delta[\mathrm{X} / \mathrm{Fe}]$ between the chemical abundances obtained from the photometric and the spectroscopic parameters for the stars n2 and a2 (observed with both MIKE and GRACES) for the

\footnotetext{
9 Available at: http://kurucz.harvard.edu/.
}

elements available from both the data sets. We note that the differences are generally small, on the order of a few hundredth dex, and are in any case lower than $0.10 \mathrm{dex} .{ }^{10}$ The upper panel represents the differences between the chemical abundances obtained from GRACES (photometric parameters) and MIKE (spectroscopic parameters) spectra for the stars $\mathrm{n} 2$ and a2. Here the differences are larger, up to $\sim 0.20 \mathrm{dex}$. The arrows displays the amount and direction of the variation in each species due to the different sets of atmospheric parameters. We note that even if in a few cases, e.g., $\mathrm{Si}$, the set of atmospheric parameters can explain the differences in the inferred abundances, in most cases the distinct results have simply to be ascribed to the different data sets and to the different spectral lines used in the analysis.

In addition to the contribution introduced by internal errors in atmospheric parameters, we estimated the contribution due to the limits of our spectra, e.g., due to the finite $\mathrm{S} / \mathrm{N}$ that affects the measurements of EWs and the spectral synthesis. For MIKE spectra, the uncertainty in the EW measurements has been estimated to be $\pm 4.4 \mathrm{~mA}$ by comparing the observed distribution of the differences between the Fe line EWs for the two MIKE stars and the corresponding distribution expected from simulated spectra (see Section 5.1). For GRACES spectra we have compared the EWs obtained from individual exposures of the same stars and obtained typical errors in EWs of 5 and $7 \mathrm{~mA}$ for the two normal and the two anomalous stars (the latter having lower $\mathrm{S} / \mathrm{N}$ ), respectively. For each spectrum, the errors in chemical abundances due to the EWs have been calculated by varying the EWs of spectral lines by the corresponding uncertainty. For the species inferred from spectral synthesis we have varied the continuum at the $\pm 1 \sigma$ level, and rederived the chemical abundances from each line.

\footnotetext{
${ }^{10}$ Iron is not included in this plot because we treat it separately in Section 5.1.
} 
Table 6

Sensitivity of the Derived Abundances to the Uncertainties in Atmospheric Parameters and Uncertainties Due to the Errors in the EWs Measurements or in the $\chi$-square Fitting Procedure

\begin{tabular}{|c|c|c|c|c|c|c|}
\hline & $\begin{array}{c}\Delta T_{\text {eff }} \\
\pm 50 \mathrm{~K}\end{array}$ & $\begin{array}{r}\Delta \log g \\
\pm 0.20\end{array}$ & $\begin{array}{c}\Delta \xi_{\mathrm{t}} \\
\pm 0.20 \mathrm{~km} \mathrm{~s}^{-1}\end{array}$ & $\begin{array}{c}\Delta[\mathrm{A} / \mathrm{H}] \\
\pm 0.10 \mathrm{dex}\end{array}$ & $\sigma_{\mathrm{EW} / \mathrm{fit}}$ & $\sigma_{\text {total }}$ \\
\hline \multicolumn{7}{|c|}{ MIKE } \\
\hline$[\mathrm{C} / \mathrm{Fe}]$ & \pm 0.07 & \pm 0.00 & \pm 0.00 & $\mp 0.03$ & \pm 0.06 & \pm 0.10 \\
\hline$[\mathrm{O} / \mathrm{Fe}]$ & \pm 0.00 & \pm 0.07 & $\mp 0.00$ & \pm 0.03 & \pm 0.09 & \pm 0.12 \\
\hline$[\mathrm{Na} / \mathrm{Fe}]$ & $\mp 0.01$ & $\mp 0.01$ & \pm 0.03 & $\mp 0.01$ & \pm 0.04 & \pm 0.05 \\
\hline$[\mathrm{Mg} / \mathrm{Fe}]$ & $\mp 0.01$ & $\mp 0.02$ & $\mp 0.01$ & \pm 0.00 & \pm 0.07 & \pm 0.07 \\
\hline$[\mathrm{Al} / \mathrm{Fe}]$ & \pm 0.05 & $\mp 0.00$ & \pm 0.01 & \pm 0.00 & \pm 0.14 & \pm 0.15 \\
\hline$[\mathrm{Si} / \mathrm{Fe}]$ & $\mp 0.07$ & \pm 0.01 & \pm 0.06 & \pm 0.02 & \pm 0.03 & \pm 0.10 \\
\hline$[\mathrm{Ca} / \mathrm{Fe}]$ & \pm 0.00 & $\mp 0.01$ & $\mp 0.01$ & $\mp 0.01$ & \pm 0.02 & \pm 0.03 \\
\hline$[\mathrm{Sc} / \mathrm{Fe}] \mathrm{II}$ & \pm 0.05 & $\mp 0.00$ & \pm 0.02 & $\mp 0.00$ & \pm 0.04 & \pm 0.07 \\
\hline$[\mathrm{Ti} / \mathrm{Fe}] \mathrm{I}$ & \pm 0.04 & $\mp 0.01$ & \pm 0.01 & $\mp 0.02$ & \pm 0.02 & \pm 0.05 \\
\hline$[\mathrm{Ti} / \mathrm{Fe}] \mathrm{II}$ & \pm 0.04 & $\mp 0.01$ & $\mp 0.04$ & $\mp 0.01$ & \pm 0.03 & \pm 0.07 \\
\hline$[\mathrm{V} / \mathrm{Fe}]$ & \pm 0.05 & $\mp 0.00$ & \pm 0.04 & $\mp 0.01$ & \pm 0.02 & \pm 0.07 \\
\hline$[\mathrm{Cr} / \mathrm{Fe}] \mathrm{I}$ & \pm 0.03 & $\mp 0.01$ & $\mp 0.00$ & $\mp 0.01$ & \pm 0.03 & \pm 0.04 \\
\hline$[\mathrm{Cr} / \mathrm{Fe}] \mathrm{II}$ & \pm 0.00 & $\mp 0.00$ & \pm 0.02 & $\mp 0.01$ & \pm 0.10 & \pm 0.10 \\
\hline$[\mathrm{Mn} / \mathrm{Fe}]$ & \pm 0.08 & \pm 0.00 & $\mp 0.02$ & $\mp 0.02$ & \pm 0.05 & \pm 0.10 \\
\hline$[\mathrm{Fe} / \mathrm{H}] \mathrm{I}$ & \pm 0.06 & \pm 0.00 & $\mp 0.07$ & $\mp 0.00$ & \pm 0.01 & \pm 0.09 \\
\hline$[\mathrm{Fe} / \mathrm{H}] \mathrm{II}$ & $\mp 0.05$ & \pm 0.07 & $\mp 0.06$ & \pm 0.04 & \pm 0.03 & \pm 0.11 \\
\hline$[\mathrm{Co} / \mathrm{Fe}]$ & \pm 0.00 & \pm 0.00 & \pm 0.06 & \pm 0.01 & \pm 0.10 & \pm 0.12 \\
\hline$[\mathrm{Ni} / \mathrm{Fe}]$ & $\mp 0.01$ & \pm 0.01 & \pm 0.04 & \pm 0.01 & \pm 0.02 & \pm 0.05 \\
\hline$[\mathrm{Cu} / \mathrm{Fe}]$ & \pm 0.08 & $\mp 0.00$ & $\mp 0.07$ & \pm 0.00 & $\mp 0.06$ & \pm 0.12 \\
\hline$[\mathrm{Zn} / \mathrm{Fe}]$ & $\mp 0.10$ & \pm 0.03 & \pm 0.01 & \pm 0.03 & \pm 0.08 & \pm 0.14 \\
\hline$[\mathrm{Sr} / \mathrm{Fe}] \mathrm{II}$ & \pm 0.09 & $\mp 0.01$ & $\mp 0.06$ & $\mp 0.03$ & \pm 0.08 & \pm 0.14 \\
\hline$[\mathrm{Y} / \mathrm{Fe}] \mathrm{II}$ & \pm 0.00 & \pm 0.05 & $\mp 0.09$ & \pm 0.00 & \pm 0.04 & \pm 0.11 \\
\hline$[\mathrm{Zr} / \mathrm{Fe}] \mathrm{II}$ & \pm 0.02 & \pm 0.06 & $\mp 0.02$ & \pm 0.01 & \pm 0.06 & \pm 0.09 \\
\hline$[\mathrm{Ba} / \mathrm{Fe}] \mathrm{II}$ & \pm 0.01 & \pm 0.07 & $\mp 0.13$ & \pm 0.03 & \pm 0.04 & \pm 0.16 \\
\hline$[\mathrm{La} / \mathrm{Fe}] \mathrm{II}$ & \pm 0.01 & \pm 0.07 & $\mp 0.02$ & \pm 0.03 & \pm 0.03 & \pm 0.08 \\
\hline$[\mathrm{Ce} / \mathrm{Fe}] \mathrm{II}$ & \pm 0.00 & \pm 0.05 & $\mp 0.05$ & \pm 0.02 & \pm 0.04 & \pm 0.08 \\
\hline$[\mathrm{Pr} / \mathrm{Fe}] \mathrm{II}$ & \pm 0.00 & \pm 0.06 & \pm 0.00 & \pm 0.04 & \pm 0.09 & \pm 0.12 \\
\hline$[\mathrm{Nd} / \mathrm{Fe}] \mathrm{II}$ & \pm 0.02 & \pm 0.06 & $\mp 0.04$ & \pm 0.01 & \pm 0.02 & \pm 0.08 \\
\hline$[\mathrm{Sm} / \mathrm{Fe}] \mathrm{II}$ & \pm 0.01 & \pm 0.06 & $\mp 0.03$ & \pm 0.01 & \pm 0.02 & \pm 0.07 \\
\hline$[\mathrm{Eu} / \mathrm{Fe}] \mathrm{II}$ & $\mp 0.01$ & \pm 0.07 & \pm 0.00 & \pm 0.04 & \pm 0.09 & \pm 0.12 \\
\hline$[\mathrm{Dy} / \mathrm{Fe}] \mathrm{II}$ & \pm 0.03 & \pm 0.08 & $\mp 0.06$ & $\mp 0.01$ & \pm 0.08 & \pm 0.13 \\
\hline \multicolumn{7}{|c|}{ GRACES Normal Stars } \\
\hline$[\mathrm{O} / \mathrm{Fe}]$ & \pm 0.01 & \pm 0.07 & \pm 0.00 & \pm 0.04 & \pm 0.14 & \pm 0.16 \\
\hline$[\mathrm{Na} / \mathrm{Fe}]$ & $\mp 0.01$ & $\mp 0.02$ & \pm 0.04 & $\mp 0.01$ & \pm 0.05 & \pm 0.07 \\
\hline$[\mathrm{Mg} / \mathrm{Fe}]$ & $\mp 0.01$ & $\mp 0.03$ & \pm 0.01 & $\mp 0.00$ & \pm 0.08 & \pm 0.09 \\
\hline$[\mathrm{Al} / \mathrm{Fe}]$ & \pm 0.03 & $\mp 0.00$ & \pm 0.00 & \pm 0.00 & \pm 0.18 & \pm 0.18 \\
\hline$[\mathrm{Si} / \mathrm{Fe}]$ & $\mp 0.09$ & \pm 0.01 & \pm 0.06 & \pm 0.02 & \pm 0.06 & \pm 0.13 \\
\hline$[\mathrm{Ca} / \mathrm{Fe}]$ & \pm 0.00 & $\mp 0.02$ & $\mp 0.01$ & $\mp 0.01$ & \pm 0.02 & \pm 0.03 \\
\hline$[\mathrm{Sc} / \mathrm{Fe}] \mathrm{II}$ & \pm 0.01 & $\mp 0.00$ & \pm 0.00 & $\mp 0.02$ & \pm 0.04 & \pm 0.05 \\
\hline$[\mathrm{Ti} / \mathrm{Fe}] \mathrm{I}$ & \pm 0.05 & $\mp 0.01$ & \pm 0.00 & $\mp 0.02$ & \pm 0.02 & \pm 0.06 \\
\hline$[\mathrm{Ti} / \mathrm{Fe}] \mathrm{II}$ & \pm 0.01 & $\mp 0.01$ & $\mp 0.06$ & $\mp 0.02$ & \pm 0.06 & \pm 0.09 \\
\hline$[\mathrm{V} / \mathrm{Fe}]$ & \pm 0.07 & $\mp 0.00$ & \pm 0.07 & $\mp 0.01$ & \pm 0.03 & \pm 0.10 \\
\hline$[\mathrm{Cr} / \mathrm{Fe}] \mathrm{I}$ & \pm 0.05 & $\mp 0.01$ & $\mp 0.04$ & $\mp 0.02$ & \pm 0.04 & \pm 0.08 \\
\hline$[\mathrm{Cr} / \mathrm{Fe}] \mathrm{II}$ & $\mp 0.02$ & $\mp 0.00$ & \pm 0.03 & $\mp 0.02$ & \pm 0.11 & \pm 0.12 \\
\hline$[\mathrm{Mn} / \mathrm{Fe}]$ & \pm 0.09 & $\mp 0.00$ & $\mp 0.02$ & $\mp 0.02$ & \pm 0.05 & \pm 0.11 \\
\hline$[\mathrm{Fe} / \mathrm{H}] \mathrm{I}$ & \pm 0.06 & \pm 0.00 & $\mp 0.09$ & \pm 0.00 & \pm 0.01 & \pm 0.11 \\
\hline$[\mathrm{Fe} / \mathrm{H}] \mathrm{II}$ & $\mp 0.04$ & \pm 0.06 & $\mp 0.07$ & \pm 0.05 & \pm 0.04 & \pm 0.12 \\
\hline$[\mathrm{Co} / \mathrm{Fe}]$ & \pm 0.01 & \pm 0.00 & \pm 0.07 & \pm 0.01 & \pm 0.09 & \pm 0.11 \\
\hline$[\mathrm{Ni} / \mathrm{Fe}]$ & $\mp 0.01$ & \pm 0.01 & \pm 0.03 & \pm 0.01 & \pm 0.03 & \pm 0.05 \\
\hline$[\mathrm{Cu} / \mathrm{Fe}]$ & \pm 0.10 & \pm 0.00 & $\mp 0.07$ & $\mp 0.02$ & \pm 0.09 & \pm 0.15 \\
\hline$[\mathrm{Y} / \mathrm{Fe}] \mathrm{II}$ & \pm 0.02 & \pm 0.04 & $\mp 0.08$ & $\mp 0.01$ & \pm 0.11 & \pm 0.14 \\
\hline$[\mathrm{Ba} / \mathrm{Fe}] \mathrm{II}$ & \pm 0.01 & \pm 0.07 & $\mp 0.15$ & \pm 0.04 & \pm 0.07 & \pm 0.18 \\
\hline$[\mathrm{La} / \mathrm{Fe}] \mathrm{II}$ & \pm 0.01 & \pm 0.06 & $\mp 0.01$ & \pm 0.04 & \pm 0.07 & \pm 0.10 \\
\hline$[\mathrm{Ce} / \mathrm{Fe}] \mathrm{II}$ & $\mp 0.02$ & \pm 0.04 & $\mp 0.06$ & \pm 0.02 & \pm 0.20 & \pm 0.21 \\
\hline$[\mathrm{Pr} / \mathrm{Fe}] \mathrm{II}$ & \pm 0.00 & \pm 0.08 & \pm 0.00 & \pm 0.04 & \pm 0.20 & \pm 0.22 \\
\hline$[\mathrm{Nd} / \mathrm{Fe}] \mathrm{II}$ & \pm 0.00 & \pm 0.06 & $\mp 0.04$ & \pm 0.02 & \pm 0.06 & \pm 0.10 \\
\hline$[\mathrm{Sm} / \mathrm{Fe}] \mathrm{II}$ & $\mp 0.01$ & \pm 0.03 & $\mp 0.05$ & \pm 0.00 & \pm 0.20 & \pm 0.21 \\
\hline
\end{tabular}


Table 6

(Continued)

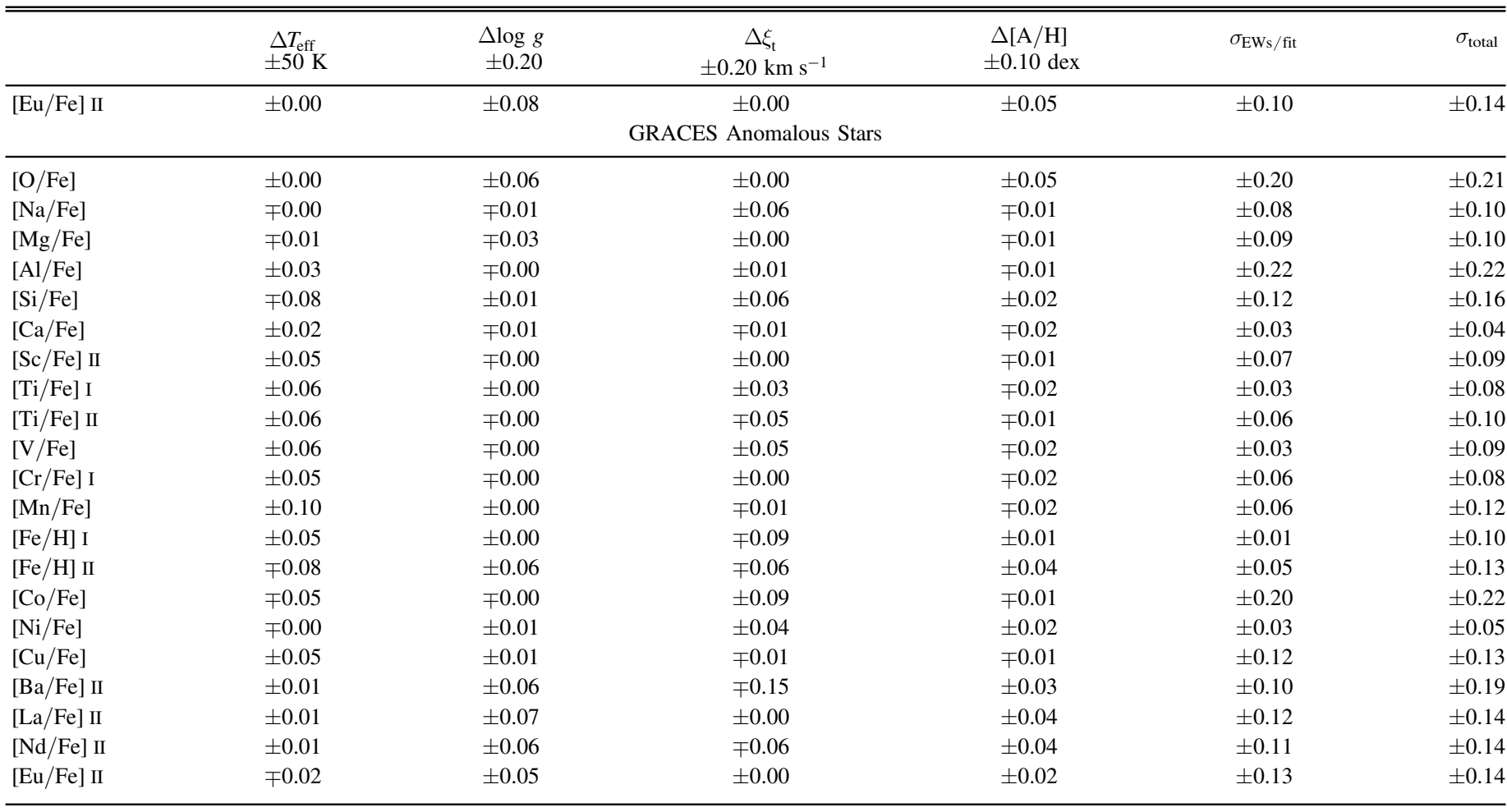

Note. We reported the total internal uncertainty $\left(\sigma_{\text {total }}\right)$ obtained by the quadratic sum of all the contributers to the error.

Since the EW/continuum placement errors are random, the error associated with elements that have a larger number of lines is lower. Hence, the corresponding uncertainty associated with Fe I is negligible ( $0.01 \mathrm{dex})$, while for the species that are inferred from one or two spectral lines, the error due to the limited $\mathrm{S} / \mathrm{N}$ is dominant (e.g., $\mathrm{Al}$, or $\mathrm{Ce}$ and $\mathrm{Pr}$ for GRACES spectra). All the error estimates are listed in Table 6 for both the MIKE spectra and the GRACES spectra, separately in the latter case for the normal and anomalous stars.

\section{The Chemical Composition of NGC 6934}

The mean metallicity obtained from our stars in NGC 6934 is $[\mathrm{Fe} / \mathrm{H}]=-1.44 \pm 0.13 \mathrm{dex}(\mathrm{rms}=0.13 \mathrm{dex})$ from the two MIKE stars (spectroscopic parameters), and $[\mathrm{Fe} / \mathrm{H}]=-1.37 \pm$ 0.12 dex $(\mathrm{rms}=0.12 \mathrm{dex})$ from the four stars observed with GRACES (photometric parameters). Figure 4 shows a summary of the other chemical abundance ratios obtained for the two stars analyzed with MIKE (upper panel), and for the four stars analyzed with GRACES (lower panels).

As is typical for Population II, the NGC 6934 stars are $\alpha$-enhanced. More specifically, the chemical abundances relative to $\mathrm{Fe}$ for the stars on the normal and anomalous RGB are the same within the observational errors for all element groups, i.e., $\alpha$, light, Fe-peak, and $n$-capture elements. In the next few subsections we consider and discuss all the interesting abundance patterns we observe in NGC 6934, focusing on those elements that play an important role in the phenomenon of multiple stellar populations. We start with iron, discuss the $p$-capture elements, and finally the $n$-process elements.

\subsection{Iron Abundances}

Even though our sample of stars is small, the results immediately suggest the presence of Fe internal variations in NGC 6934. From a simple comparison between the spectra of the normal and anomalous stars $\mathrm{n} 2$ and a2, which have very similar atmospheric parameters, it is clear that a2 displays stronger lines, as shown in Figure 5. On the other hand, the hydrogen lines $(\mathrm{H} \alpha$ and $\mathrm{H} \beta$ ) are very similar, confirming that the stars have the same effective temperature.

Figure 6 displays the Fe I and Fe II abundances as a function of $\Delta_{\mathrm{F} 275 \mathrm{~W}, \mathrm{~F} 814 \mathrm{~W}}$ for the analyzed stars for both the MIKE and GRACES spectra. We note the systematic higher Fe abundances for the GRACES data, as discussed in Section 3, but to avoid these systematic differences in our discussion, we refer to the relative Fe differences observed in each data set separately.

In particular, the iron abundances, both neutral and singly ionized, are higher in the stars located on the anomalous RGB. This remains valid independent of which set of atmospheric parameters are used, spectroscopic or photometric. These anomalous stars also have higher $\Delta_{\mathrm{F} 275 \mathrm{~W}, \mathrm{~F} 814 \mathrm{~W}}$ values than normal RGB stars in the chromosome map.

In all the panels, two error bars have been associated with each Fe measurement: the error defined as $\mathrm{rms} / \sqrt{(N-1)}$, where $N$ is the number of lines (gray error bars); and the expected error due to the model atmospheres and the $\mathrm{S} / \mathrm{N}$ (black error bars, as discussed in Section 4). The larger number of available Fe I lines (upper panels) translates into a statistical error rms $/ \sqrt{(N-1)}$ smaller than the one associated with Fe II (lower panels). The expected errors listed in Table 6 are larger, ranging between 0.09 and 0.11 for $\mathrm{Fe} \mathrm{I}$, and between 0.11 to 0.13 for Fe II. 

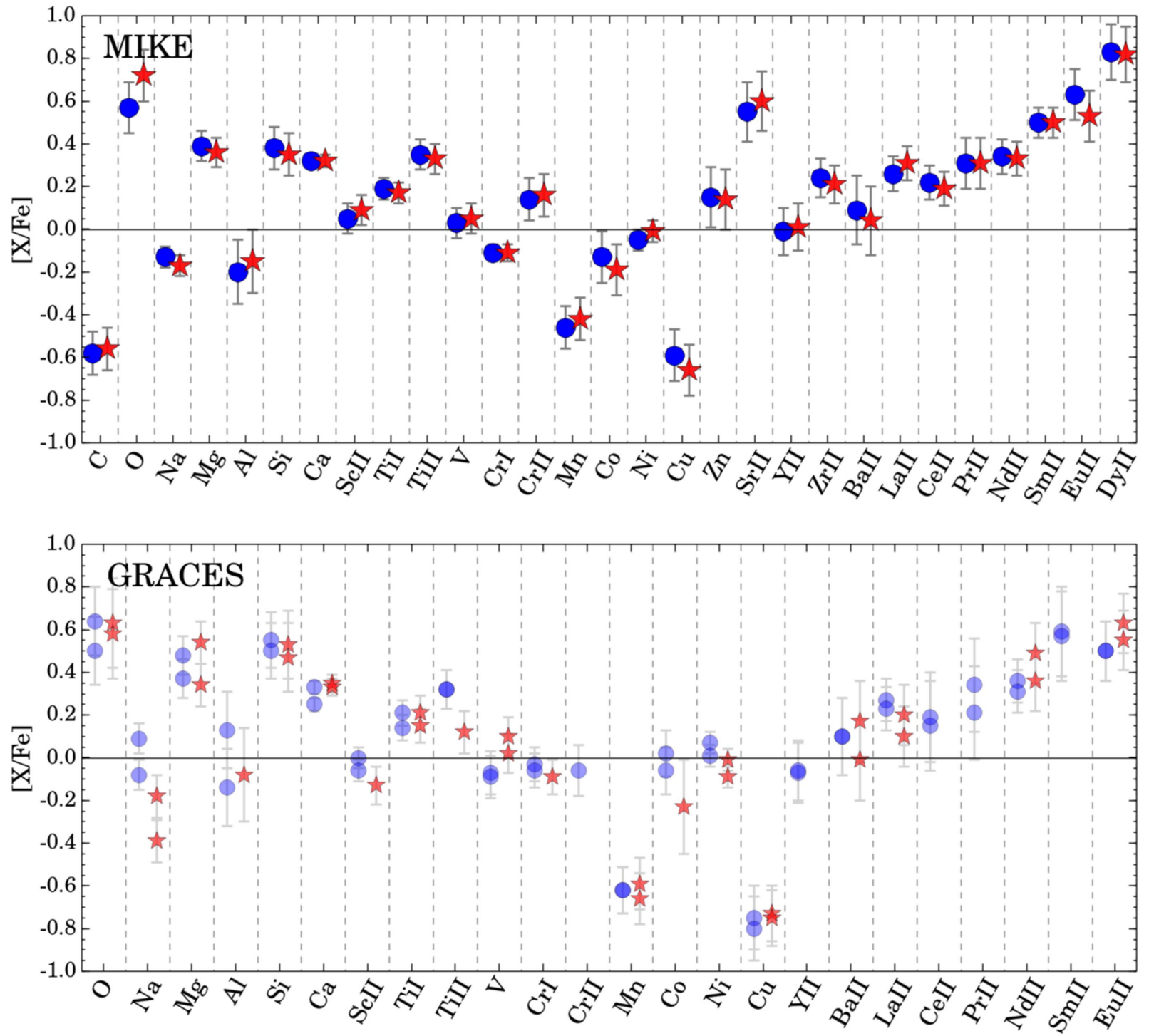

Figure 4. Summary of the abundance ratios results obtained from the MIKE (upper panel) and GRACES (lower panel) spectra. For each species, we plot the [X/Fe] relative abundances. In both panels, red stars are used for stars located on the red sequence of the chromosome map, and filled blue circles denote stars on the normal sequence.

Quantitatively, we obtain $\Delta[\mathrm{Fe} \mathrm{I} / \mathrm{H}]=0.18 \pm 0.13$ dex for MIKE (by construction, $\Delta[\mathrm{Fe} \mathrm{II} / \mathrm{H}]$ is the same, but has a larger error of \pm 0.16 ); and $\Delta[\mathrm{Fe} \mathrm{I} / \mathrm{H}]=0.21 \pm 0.15 \mathrm{dex}$ and $\Delta[\mathrm{Fe}$ II $/ \mathrm{H}]=0.23 \pm 0.18 \mathrm{dex}$ for GRACES. The weighted averages of the two normal and anomalous stars observed with GRACES are $\langle[\mathrm{Fe} \mathrm{I} / \mathrm{H}]\rangle=-1.47 \pm 0.08 \mathrm{dex}$ and $\langle[\mathrm{Fe} \mathrm{I} / \mathrm{H}]\rangle=$ $-1.26 \pm 0.07 \mathrm{dex}$, respectively, which means $\Delta[\mathrm{Fe} \mathrm{I} / \mathrm{H}]=$ $0.21 \pm 0.11$ dex. By taking advantage of the measurements from both MIKE and GRACES, the weighted $\Delta[\mathrm{Fe} \mathrm{I} / \mathrm{H}]$ mean value is $\Delta[\mathrm{Fe} \mathrm{I} / \mathrm{H}]=0.20 \pm 0.08 \mathrm{dex}$, which is a significance of $\sim 2.5 \sigma$.

Although the small observed sample prevents us from drawing strong conclusions on the higher $\mathrm{Fe}$ abundance for the anomalous RGB stars, the Fe enrichment is observed in all our available measurements, independent of the set of the adopted stellar parameters and/or data. Thus, it is very tempting to conclude that the anomalous stars are enriched in the overall metallicity. In the following we present a few independent tests to corroborate the presence of Fe variations in NGC 6934.

First, in Figure 7 we show the difference in EWs for the $\mathrm{Fe}$ lines between the two stars observed with MIKE (a2-n2). The observed differences have been compared with those expected for two simulated spectra with the same atmospheric parameters of the two target stars. We note that while the observed mean difference for $\mathrm{Fe} \mathrm{I}$ is relatively high, $9.9 \mathrm{~m} \AA$, that for the Fe II is smaller, $2.9 \mathrm{~m} \AA$. In both cases the observed differences agree well with those expected from theoretical spectra $(\Delta$ EWs $(F e \mathrm{I})=10.4 \mathrm{~m} \AA$ and $\Delta$ EWs $(F e ~ I I)=1.2 \mathrm{~m} \AA$ ). The small difference in the Fe II lines is well explained by the atmospheric parameters of our stars. Indeed, we obtain higher surface gravities for the two stars that are located on the anomalous sequence of the chromosome map. Assuming that all the other atmospheric parameters are identical, a star with higher $\log g$ is additionally expected to show smaller EWs.

Second, an independent test that provides further evidence for iron variations in NGC 6934 comes from the comparison between the observed CMD and stellar isochrones. Figure 8 represents the $m_{\mathrm{F} 336 \mathrm{~W}}-\left(m_{\mathrm{F} 336 \mathrm{~W}}-m_{\mathrm{F} 814 \mathrm{~W}}\right)$ and $m_{\mathrm{F} 438 \mathrm{~W}^{-}}\left(m_{\mathrm{F} 438 \mathrm{~W}}-m_{\mathrm{F} 606 \mathrm{~W}}\right) \mathrm{CMDs}$ for the cluster. The presence of an additional sequence, on the red side of the main RGB, is clear in the $m_{\mathrm{F} 336 \mathrm{~W}}-\left(m_{\mathrm{F} 336 \mathrm{~W}}-m_{\mathrm{F} 814 \mathrm{~W}}\right)$ 

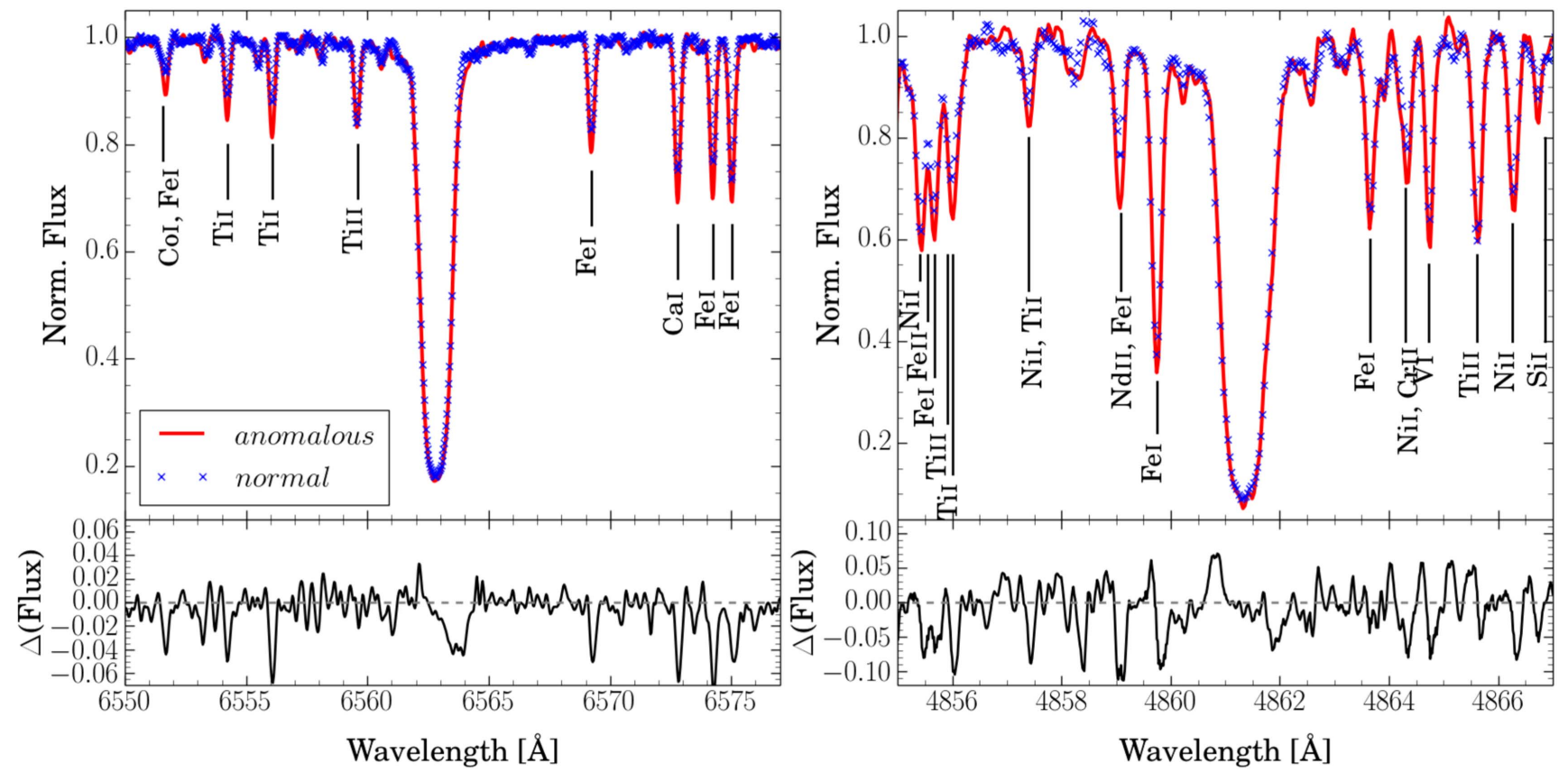

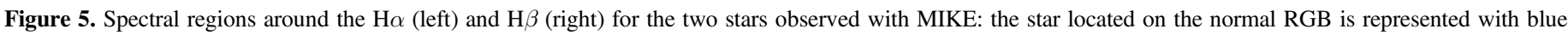

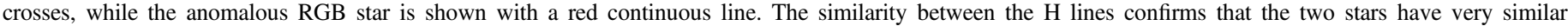
atmospheric parameters. The difference between the normalized fluxes of the two stars is displayed in the lower panels.

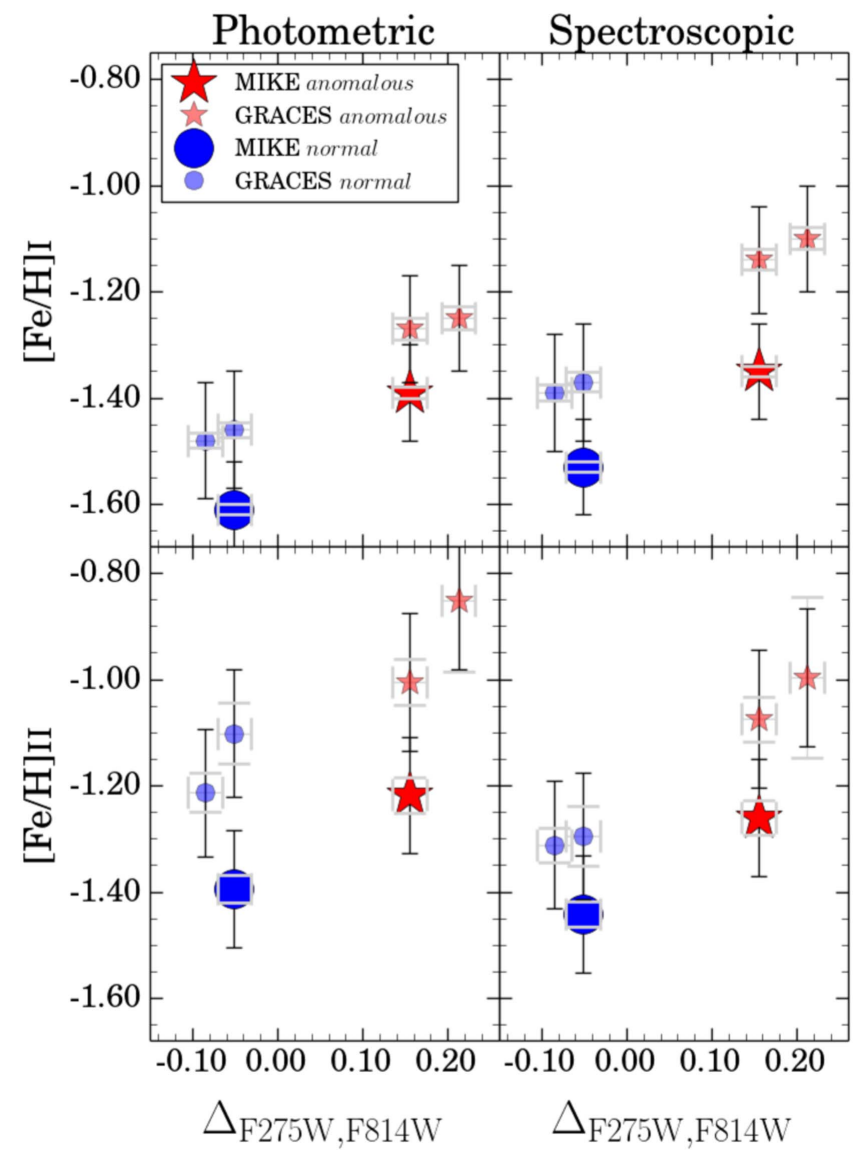

Figure 6. Chemical abundances obtained for Fe I and Fe II by using atmospheric parameters derived from Fe spectral lines (right panels) and from photometry (left panels). Stars in the normal and red sequence of the chromosome map have been plotted with blue circles and red stars, respectively. Results obtained from MIKE and GRACES are distinguished by larger darker symbols for MIKE and smaller lighter symbols for GRACES.
CMD. Stars in the anomalous RGB, which evolve from a fainter SGB, correspond to the stars defining the anomalous sequence on the chromosome map (on Figure 1), and are also located redder in the $m_{\mathrm{F} 438 \mathrm{~W}}-\left(m_{\mathrm{F} 438 \mathrm{~W}}-m_{\mathrm{F} 606 \mathrm{~W}}\right)$ CMD. Superimposed on both CMDs are isochrones corresponding to an age $=12.25 \mathrm{Gyr}$, $Y=0.2471,[\alpha / \mathrm{Fe}]=+0.40$, and $[\mathrm{Fe} / \mathrm{H}]=-1.40$ (dark red) and $[\mathrm{Fe} / \mathrm{H}]=-1.60$ (black), retrieved from the Dartmouth database (Dotter et al. 2008). Although the upper part of the RGB is poorly represented by these isochrones, especially in the $m_{\mathrm{F} 336 \mathrm{~W}^{-}}$ $\left(m_{\mathrm{F} 336 \mathrm{~W}}-m_{\mathrm{F} 814 \mathrm{~W}}\right) \mathrm{CMD}$ (possibly due to the contribution of CNO abundances to $m_{\mathrm{F} 336 \mathrm{~W}}$ ), the fit on the lower RGB and the SGB is satisfactory, with only some systematic shift of both isochrones on the RGB blue side. We conclude that a difference in $[\mathrm{Fe} / \mathrm{H}]$ by $\sim 0.2$ dex can reproduce the CMD of NGC 6934 . According to our HST high-precision CMD, NGC 6934 hosts one main stellar population, and one minor component that is slightly enriched in $\mathrm{Fe}$, and which constitutes $7 \pm 1 \%$ of the cluster mass (Milone et al. 2017).

The inset in Figure 8 shows the theoretical $\log g-T_{\text {eff }}$ plane from the Dartmouth isochrones with $[\mathrm{Fe} / \mathrm{H}]=-1.60$ and $[\mathrm{Fe} / \mathrm{H}]=-1.40$. Superimposed are our adopted atmospheric parameters for the analyzed stars, specifically, spectroscopic parameters for MIKE and photometric parameters for GRACES (see Section 3). Both sets of adopted $\log g-T_{\text {eff }}$ parameters agree with the parameters expected from theoretical isochrones and satisfy the basic principle that at a given $T_{\mathrm{eff}}$, stars with higher metallicity have higher $\log g$. Specifically, the $\log g$ difference expected at $T_{\text {eff }} \sim 4450 \mathrm{~K}$ is $\sim 0.15-0.20 \mathrm{dex}$, consistent with our estimates.

\subsection{Light Elements}

The abundance ratios obtained for the $p$-capture elements from $\mathrm{C}$ to $\mathrm{Al}$ have been plotted in the left panel of Figure 9. As a comparison, we have also included the same elements derived for M 22 by Marino et al. (2009, 2011a). A first fact to note is 

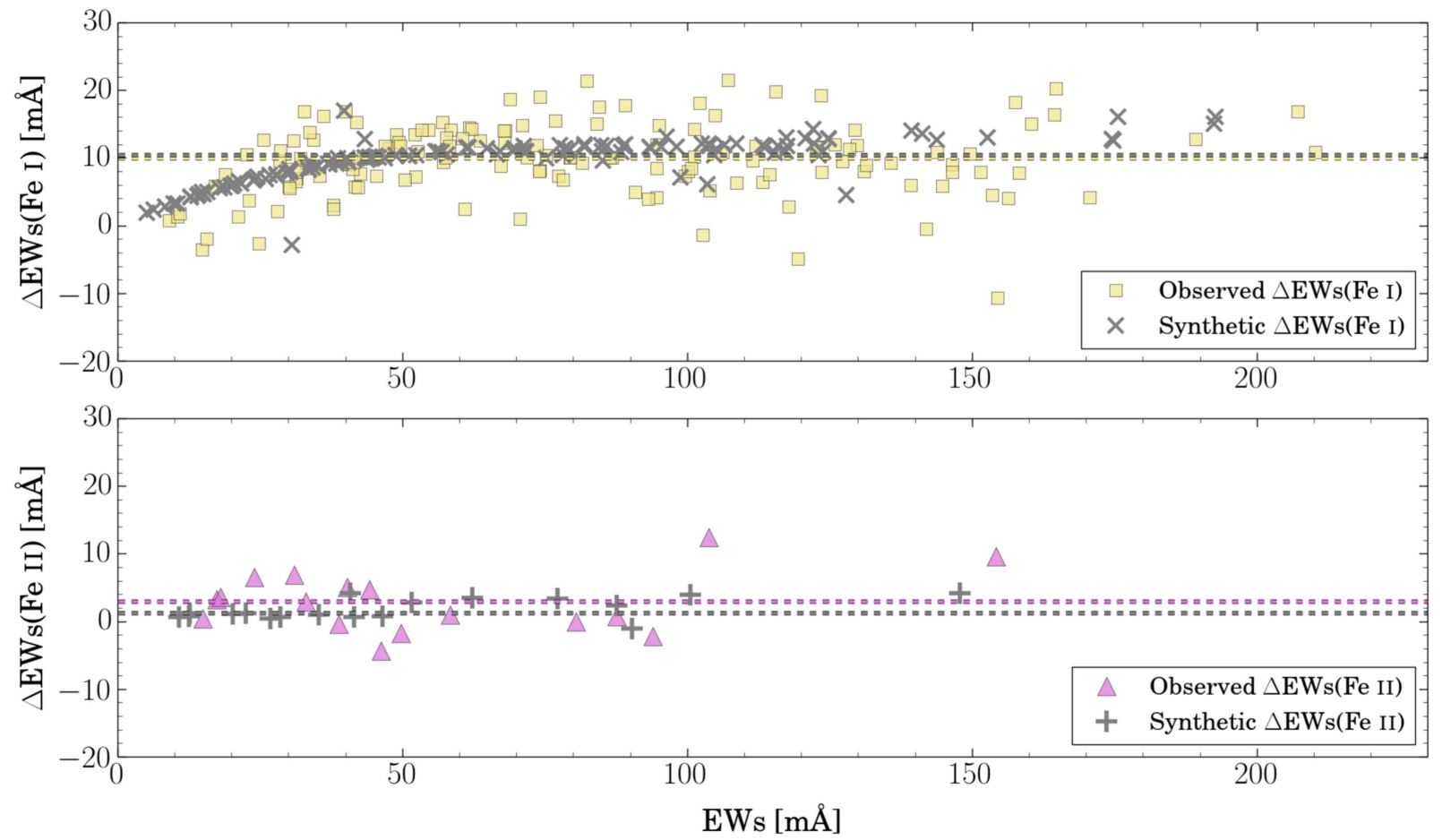

Figure 7. Observed difference in Fe I (upper panel, yellow squares) and Fe II (lower panel, magenta triangles) EWs between stars $\mathrm{n} 2$ and a2, observed with MIKE, as a function of EW. The two stars have very similar atmospheric parameters, but their $[\mathrm{Fe} / \mathrm{H}]$ differs by $\sim 0.20$ dex. Gray symbols in both panels represent the EW differences for the same Fe lines, but computed from two synthetic spectra with the same atmospheric parameters as the two program stars and a difference of 0.2 dex in $[\mathrm{Fe} / \mathrm{H}]$.
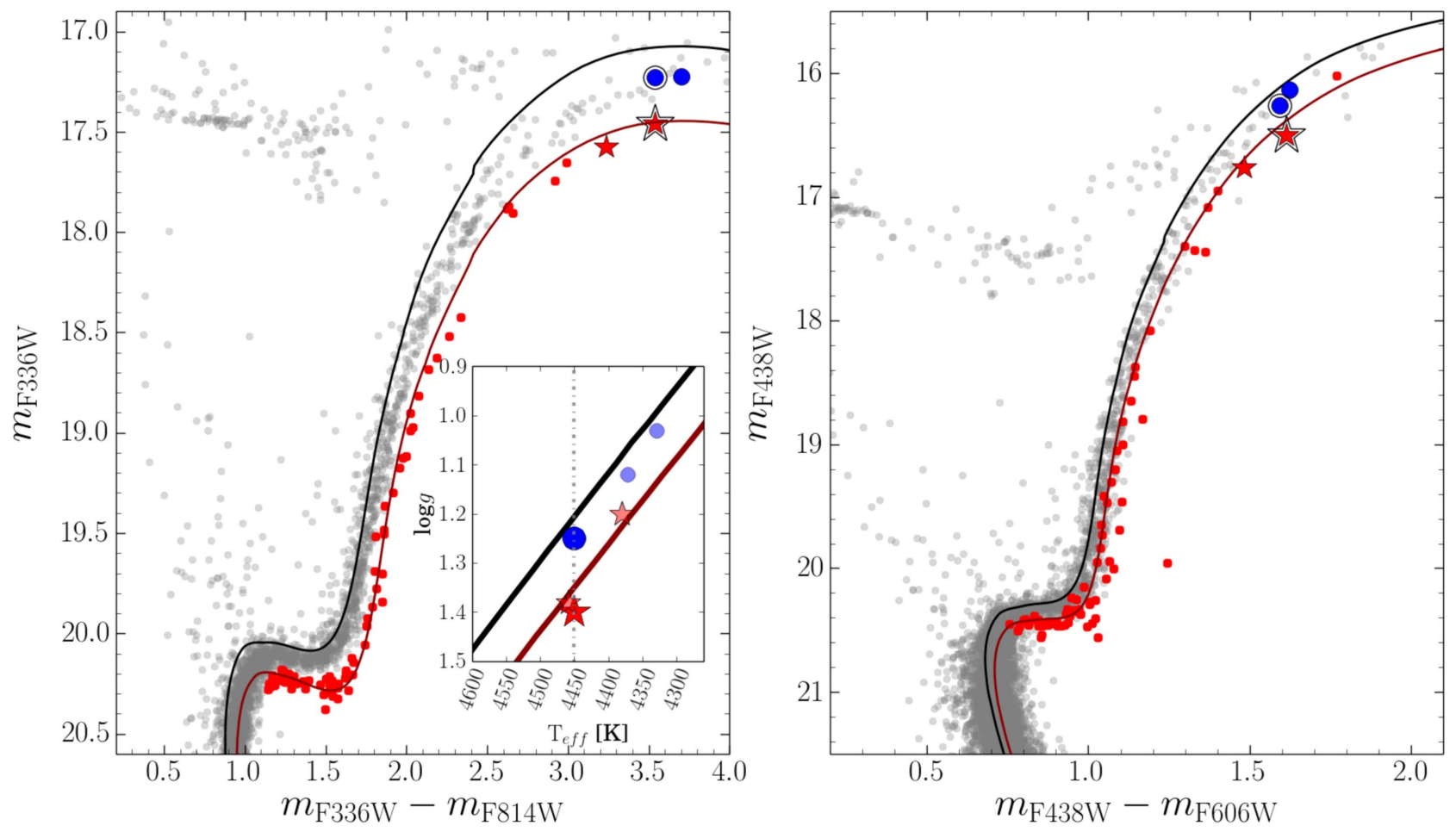

Figure 8. $m_{\mathrm{F} 336 \mathrm{~W}}$ vs. $m_{\mathrm{F} 336 \mathrm{~W}}-m_{\mathrm{F} 814 \mathrm{~W}}$ (left panel) and $m_{\mathrm{F} 438 \mathrm{~W}}$ vs. $m_{\mathrm{F} 438 \mathrm{~W}}-m_{\mathrm{F} 606 \mathrm{~W}}$ (right panel) CMDs for NGC 6934. Anomalous RGB stars and faint SGB stars have been plotted in red colors. Superimposed on the CMDs are two isochrones from the Dartmouth database (Dotter et al. 2008), with the same age (12.25 Gyr), helium $(Y=0.2471)$, and $\alpha$ content $([\alpha / \mathrm{Fe}]=+0.40)$, but different metallicity. Specifically, the black isochrone has $[\mathrm{Fe} / \mathrm{H}]=-1.60$, and the dark-red one $[\mathrm{Fe} / \mathrm{H}]=$ $-1.40 \mathrm{dex}$. The inset in the left panel shows the $\log g$ vs. $T_{\text {eff }}$ theoretical plane with the pattern corresponding to the two isochrones, together with the adopted stellar parameters for the target stars. The plotted $\log g-T_{\text {eff }}$ values have been derived spectroscopically for the MIKE data (plotted with larger symbols), and photometrically for GRACES data (plotted with smaller lighter symbols). 

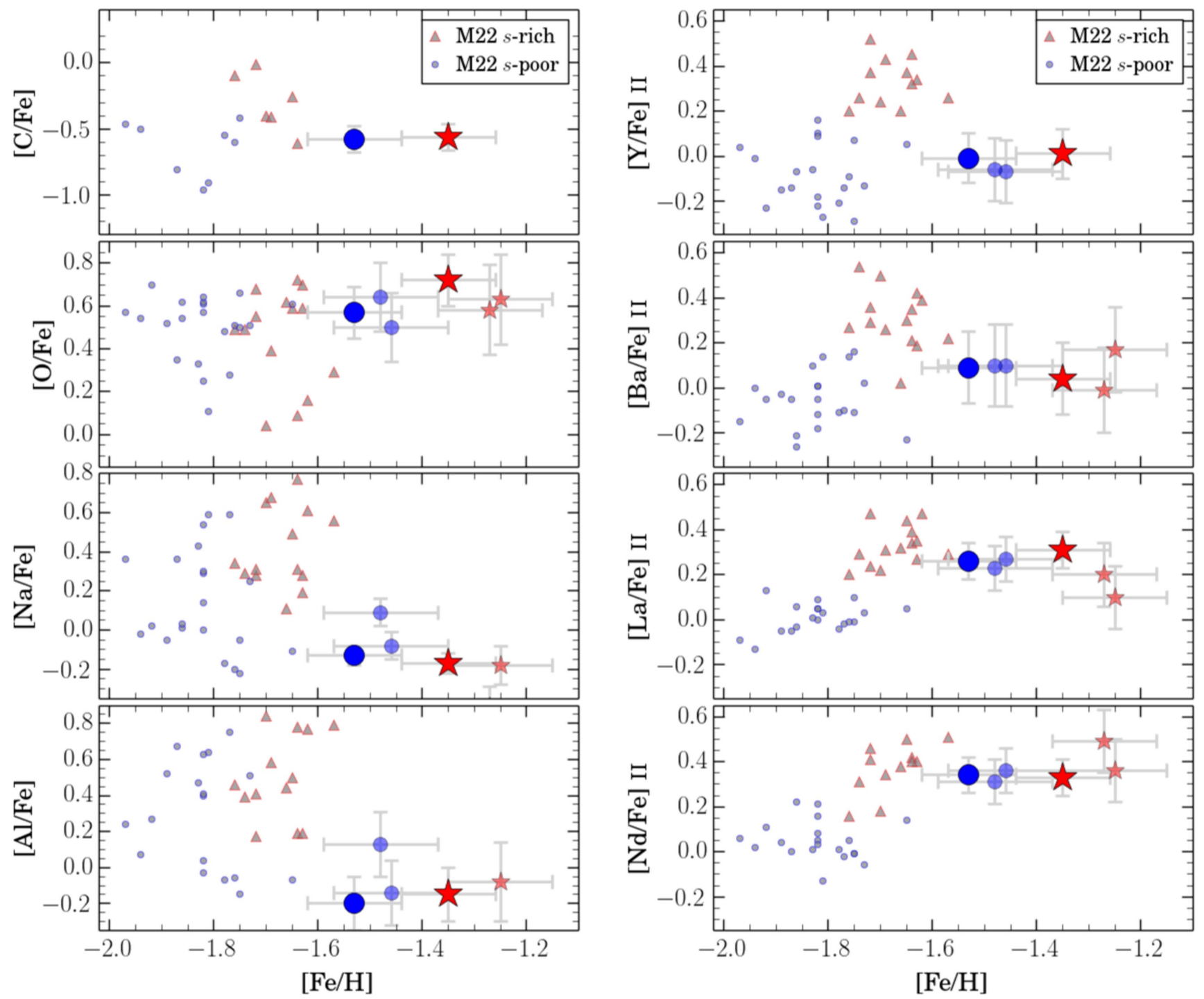

Figure 9. Abundance ratios of light elements [C-O-Na-Al/Fe] (left panel) and [Y-Ba-La-Nd/Fe] II (right panel), derived for NGC 6934 as function of [Fe/H], compared to the two $s$ groups observed in M 22. s-poor and $s$-rich stars in M 22 are plotted with small gray-blue circles and gray-red triangles, respectively. Because of the different adopted $\mathrm{O}$ solar abundance, $[\mathrm{O} / \mathrm{Fe}]$ abundances for $\mathrm{M} 22$ have been shifted by +0.14 dex. Blue circles and red stars represent stars in the normal and red sequence of the NGC 6934 chromosome map, respectively.

that the analyzed stars in NGC 6934 do not seem to show large dispersions in these elements. All our NGC 6934 targets lie in the O-rich/Na-Al-poor range, e.g., they all share a primordial chemical composition as regards the $p$-capture elements. On the other hand, both the $s$-poor and the $s$-rich stars analyzed in M 22 span a relatively large range of abundances in all these elements, including stars with chemical composition typical for second generation(s), e.g., O-depleted and Na-Al-enhanced. Carbon abundances have been inferred only for the two stars observed with MIKE, and the normal and the anomalous stars show similar values.

The lack of second-generation stars in our NGC 6934 sample is due to the selection of our targets, which is biased toward stars located in the lower part of the chromosome map with relatively low $\Delta_{C}$ F275W,F336W,F438W values. As shown in Milone et al. (2017), first population stars possess relatively low values of $\Delta_{C}$ F275W,F336W,F438W, and relatively high values of $\Delta_{\mathrm{F} 275 \mathrm{~W}, \mathrm{~F} 814 \mathrm{~W}}$. The choice to observe only stars in the lower part of the chromosome map is justified by our goal to investigate any difference (if present) between the chemical composition of stars in the normal and anomalous RGBs. To do this, we tried to avoid additional effects such as the variation in light elements within the normal and anomalous categories. We expect that second-population stars, with low $\mathrm{O}$ and high $\mathrm{Na}$, would be observed to have higher $\Delta_{C}$ F275W,F336W,F438W values. In this context, the higher $\mathrm{Na}$ and $\mathrm{Al}$ abundance ratios inferred for the normal star $\mathrm{n} 1$ agree with its position on the chromosome map, given its slightly higher $\Delta_{C}$ F275W,F336W,F438W. Future observations will clarify the light-element distribution in the normal and anomalous RGBs of NGC 6934.

\subsection{S-process Elements}

In the right panel of Figure 9 we show the abundance ratios of some of the analyzed $n$-capture elements, specifically, Y, Ba, $\mathrm{La}$, and $\mathrm{Nd}$ relative to $\mathrm{Fe}$, as a function of $[\mathrm{Fe} / \mathrm{H}]$ both from MIKE and GRACES spectra. As done for the light elements, in each panel we plot the corresponding results obtained for M 22 (data from Marino et al. 2009 and Marino et al. 2011a). 
Table 7

List of GCs with Confirmed Chemical and/or Photometric Anomalies

\begin{tabular}{|c|c|c|c|c|c|c|c|c|c|}
\hline \multirow{2}{*}{ GC } & \multicolumn{8}{|c|}{ Chemical Abundance Variations } & \multirow{2}{*}{ Proposed Class } \\
\hline & Metallicity & Literature & $s$-elements & Literature & $\begin{array}{c}p \text {-capture } \\
\text { Elements } \\
\text { in Each Fe Group }\end{array}$ & Literature & $\mathrm{C}+\mathrm{N}+\mathrm{O}$ & Literature & \\
\hline NGC 1261 & (?) & & (?) & & (?) & & (?) & & Type II \\
\hline NGC 1851 & Possible small & $\begin{array}{l}\text { Carretta et al. } \\
(2010) \\
\text { Gratton et al. } \\
(2013) \\
\text { Marino et al. } \\
(2014)\end{array}$ & Yes & $\begin{array}{l}\text { YG08 }^{\mathrm{a}} \text {; } \\
\text { Villanova et al. } \\
\quad(2010)\end{array}$ & Yes & $\begin{array}{l}\text { Carretta et al. } \\
(2010) \\
\text { Villanova et al. } \\
\quad(2010)\end{array}$ & Yes & $\begin{array}{l}\text { Yong et al. } \\
\text { (2014) }\end{array}$ & Iron-II/s-II/Type II \\
\hline NGC 362 & (?) & & Yes & Carretta et al. (2013) & (?) & & (?) & & $s$-II/Type II \\
\hline $\begin{array}{l}\text { NGC } 5139 \\
(\omega \text { Centauri) }\end{array}$ & Yes & $\begin{array}{l}\text { Norris et al. } \\
(1996) \\
\text { SK96 }^{\mathrm{d}}\end{array}$ & Yes & $\begin{array}{l}\text { NDaC95 } \\
\text { Smith et al. (2000) } \\
\text { JP10 } \\
\text { Marino et al. (2011b) } \\
\text { D'Orazi et al. (2011) }\end{array}$ & Yes & $\begin{array}{l}\text { JP10 } \\
\text { Marino et al. } \\
\quad(2011 \mathrm{~b})\end{array}$ & Yes & $\begin{array}{l}\text { Marino et al. } \\
(2012 \mathrm{~b})\end{array}$ & Iron-II/s-II/Type II \\
\hline NGC 5286 & Yes & $\begin{array}{l}\text { Marino et al. } \\
\text { (2015) }\end{array}$ & Yes & Marino et al. (2015) & Yes & $\begin{array}{l}\text { Marino et al. } \\
\text { (2015) }\end{array}$ & (?) & & Iron-II/s-II/Type II \\
\hline NGC 5824 & Yes(?) & $\begin{array}{l}\text { Da Costa } \\
\text { et al. (2014) }\end{array}$ & Yes(?) & $\begin{array}{l}\text { Roederer et al. } \\
\text { (2016) }\end{array}$ & (?) & & (?) & & Iron-II(?)/s-II(?) \\
\hline NGC 6229 & Possible small & $\begin{array}{l}\text { Johnson et al. } \\
\text { (2017) }\end{array}$ & Yes & Johnson et al. (2017) & No(?) & $\begin{array}{l}\text { Johnson et al. } \\
\text { (2017) }\end{array}$ & (?) & & Iron-II(?)/s-II \\
\hline NGC 6273 (M 19) & Yes & $\begin{array}{l}\text { Johnson et al. } \\
\text { (2015) }\end{array}$ & Yes & Johnson et al. (2015) & Yes & $\begin{array}{l}\text { Johnson et al. } \\
\text { (2015) }\end{array}$ & (?) & & Iron-II $/ s$-II \\
\hline NGC 6388 & (?) & & (?) & & (?) & & (?) & & Type II \\
\hline NGC 6656(M 22) & Yes & $\begin{array}{l}\text { Marino et al. } \\
(2009) \\
\text { Da Costa } \\
\text { et al. (2009) }\end{array}$ & Yes & $\begin{array}{l}\text { Marino et al. (2009, } \\
\text { 2011a, 2012a) }\end{array}$ & Yes & $\begin{array}{l}\text { Marino et al. } \\
\quad(2009,2011 \mathrm{a})\end{array}$ & Yes & $\begin{array}{l}\text { Marino et al. } \\
\text { (2011a) } \\
\text { Alves Brito } \\
\text { et al. (2012) }\end{array}$ & Iron-II/s-II/Type II \\
\hline NGC 6715 (M 54) & Yes & $\begin{array}{l}\text { Carretta et al. } \\
\text { (2010) }\end{array}$ & Yes(?) & BWG99 ${ }^{\mathrm{e}}$ & Yes & $\begin{array}{l}\text { Carretta et al. } \\
\text { (2010) }\end{array}$ & (?) & & $\overline{\text { Iron II/s-II(?)/Type II }}$ \\
\hline NGC 6934 & Yes & This work & No & This work & (?) & & (?) & & Iron II/s-I/Type II \\
\hline NGC 7089 (M 2) & Yes & $\begin{array}{l}\text { Yong et al. } \\
\text { (2014) }\end{array}$ & Yes & $\begin{array}{l}\text { Lardo et al. (2013) } \\
\text { Yong et al. (2014) }\end{array}$ & Yes & $\begin{array}{l}\text { Yong et al. } \\
\text { (2014) }\end{array}$ & (?) & & Iron-II/s-II/Type II \\
\hline
\end{tabular}


Table 7

(Continued)

\begin{tabular}{|c|c|c|c|c|c|c|c|c|c|}
\hline \multirow{2}{*}{ GC } & \multicolumn{8}{|c|}{ Chemical Abundance Variations } & \multirow{2}{*}{ Proposed Class } \\
\hline & Metallicity & Literature & $s$-elements & Literature & $\begin{array}{c}p \text {-capture } \\
\text { Elements } \\
\text { in Each Fe Group }\end{array}$ & Literature & $\mathrm{C}+\mathrm{N}+\mathrm{O}$ & Literature & \\
\hline Terzan 5 & Yes & $\begin{array}{l}\text { Ferraro et al. } \\
(2009) \\
\text { Origlia et al. } \\
(2011) \\
\text { Massari et al. } \\
\text { (2014) }\end{array}$ & (?) & & (?) & & (?) & & Iron-II \\
\hline
\end{tabular}

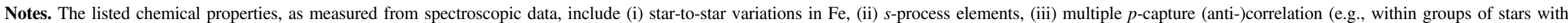

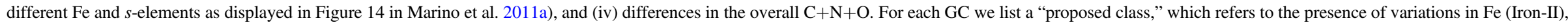
in $s$-elements (s-II), and multiple sequence on the chromosome map, as found in Milone et al. (2017, Type II). Further details are given in Section 6.

a Yong \& Grundahl (2008).

b Norris \& Da Costa (1995).

${ }^{\mathrm{c}}$ Johnson \& Pilachowski (2010).

${ }^{\mathrm{d}}$ Suntzeff \& Kraft (1996).

e Brown et al. (1999). 
Although it is a more metal-poor GC, M 22 has been chosen for comparison purposes because it shows a clear bimodality in $n$-capture elements. Typically, in anomalous GCs such as M 22, the variation in $n$-capture elements is due to additional $s$-process enrichment among some second-generation stars (Marino et al. 2011a). Specifically, M 22 hosts a stellar population that is relatively enriched in the $s$-process elements ( $s$-rich group) with respect to a stellar population with lower $s$ elements abundance ratios ( $s$-poor group). The $s$-rich group is also enhanced in $\mathrm{Fe}$ by $\sim 0.15$ dex and in the overall $[\mathrm{C}+\mathrm{N}$ $+\mathrm{O} / \mathrm{Fe}$ ] (Marino et al. 2009, Marino et al. 2011a, Marino et al. 2012a). However, the $n$-capture element $[\mathrm{Eu} / \mathrm{Fe}]$ ratio, which is mostly produced via rapid neutron-capture reactions ( $r$-process element), is uniform.

We note that the difference in Fe between the $s$-rich and the $s$-poor groups of M 22 is similar to the measured difference between the normal and anomalous stars in NGC 6934. However, while for M 22 the variation in Fe is coupled with a variation in $[s$-elements $/ \mathrm{Fe}]$, with the Fe-richer stars displaying higher $[s$-elements $/ \mathrm{Fe}]$, the analyzed stars in NGC 6934 exhibit similar content for $\mathrm{Y}, \mathrm{Ba}, \mathrm{La}$, and $\mathrm{Nd}$ relative to $\mathrm{Fe}$. Indeed, by considering, e.g., La, which is one of the $s$-elements with more precise measured abundances, we derive a difference of $|\Delta[\mathrm{La} / \mathrm{Fe}]|=0.05 \pm 0.11 \mathrm{dex}$ (from MIKE data) and $|\Delta[\mathrm{La} / \mathrm{Fe}]|=-0.10 \pm 0.11 \mathrm{dex}$ (from GRACES data), compared to the significantly larger difference $|\Delta[\mathrm{La} / \mathrm{Fe}]|=0.32 \pm 0.02$ dex between the $s$-rich and $s$-poor group of M 22. We recall here that M 22 displays one of the smallest internal variation in the $[s$-element $/ \mathrm{Fe}]$ ratio among the known anomalous GCs (see e.g., Figure 19 in Marino et al. 2015). For comparison, $\mathrm{M} 2$ has $|\Delta[\mathrm{La} / \mathrm{Fe}]|=0.58 \mathrm{dex}$ (Yong et al. 2014), NGC 5286 $|\Delta[\mathrm{La} / \mathrm{Fe}]|=0.56$ dex (Marino et al. 2015), and NGC 6273 $|\Delta[\mathrm{La} / \mathrm{Fe}]|=0.42 \mathrm{dex}$ (Johnson et al. 2017).

Clearly, the Type II GC NGC 6934 seems different from the chemically defined class of anomalous GCs, as our analyzed sample does not show any evidence of additional internal variations in $s$-elements. This conclusion is based only on four stars, and future observations on larger sample sizes may perhaps reveal the presence of stars enhanced in $s$-elements located in other regions of the chromosome map. On the other hand, we note that despite our sample of only four stars, they have been carefully selected from the two different sequences in the chromosome map where we expect variations in heavy elements, as observed in the anomalous GCs. Photometrically, $s$-rich stars in anomalous GCs are located on the red RGB (Milone et al. 2017). We cannot exclude a different behavior for NGC 6934, however.

An inspection of the other $n$-capture species in Figure 4 (from Sr to Dy for MIKE and from Y to Eu for GRACES) clearly suggests the lack of any significant internal variation in the analyzed elements in NGC 6934. In this figure we note a growth of the abundances relative to $\mathrm{Fe}$ as a function of the atomic number in both the normal and anomalous stars.

\section{Discussion and Conclusions}

We have presented a high-resolution chemical abundance analysis of four stars on the normal and anomalous RGB of NGC6934. We have found that the chemical abundances of all the inferred species, except the abundances of $\mathrm{Fe}$, are consistent with uniform abundance ratios in the four analyzed stars. The difference in $\mathrm{Fe}$ is on the order of $\sim 0.2$ dex with two anomalous RGB stars exhibiting higher abundances. This variation in $\mathrm{Fe}$ is corroborated by the comparison between the CMDs obtained from high-precision HST photometry and the isochrones of Dotter et al. (2008).

NGC 6934 has been classified as a Type II GC as, in contrast to Type I GCs, it displays more than the normal $1 \mathrm{G}$ and $2 \mathrm{G}$

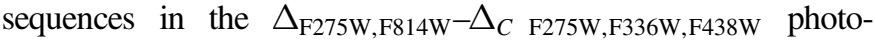
metric plane. Milone et al. (2017) have shown that the main pattern on the chromosome map in all GCs, both Type I and Type II, corresponds to a distribution in light-element abundances: stars with different $\mathrm{He}, \mathrm{C}, \mathrm{N}, \mathrm{O}$, and $\mathrm{Na}$ abundances distribute from higher $\Delta_{\mathrm{F} 275 \mathrm{~W}, \mathrm{~F} 814 \mathrm{~W}}$ and lower $\Delta_{C}$ F275W,F336W,F438W to lower $\Delta_{\mathrm{F} 275 \mathrm{~W}, \mathrm{~F} 814 \mathrm{~W}}$ and higher $\Delta_{C}$ F275W,F336W,F438W.

The presence of numerous additional sequences on the red side of the main chromosome map appears in $\sim 18 \%$ of the analyzed GCs (Type II). For a subsample of these GCs, it was possible to identify some stars with available chemical abundances along the sequences of the chromosome map, and it appeared that the additional red sequences are populated by stars enriched in both $\mathrm{Fe}$ and $s$-elements relative to $\mathrm{Fe}$ (anomalous GCs). This behavior is also observed in $\omega$ Centauri.

For simplicity, a list of the currently known chemically anomalous and/or photometrically peculiar Type II GCs is provided in Table 7. In the light of the new findings coming from the synergy between spectroscopic and photometric investigation, we propose a slighly different and simpler terminology to define this new class of GCs, as listed in the last column of the table:

1. Iron II are the objects with variations in $\mathrm{Fe}$, in contrast to the typical GCs, designated Iron I, that do not show evidence for star-to-star variations in $\mathrm{Fe}$;

2. $s$ II is used for the GCs with variations in s-elements, while typical GCs are labeled $s$-I; and

3. following Milone et al. (2017), Type II is used for the GCs with additional red sequences on the chromosome map, while typical GCs are Type I.

With this terminology, NGC 6934 can be classified as a Iron II/s-I/Type II GC. However, we cannot exclude that a future analysis of larger sample sizes may reveal stars with enhancements in the $s$-elements.

Our results show that although NGC 6934 is classified as a Type II GC, its anomalous stars do not exhibit any enrichment in the $s$-process elements relative to $\mathrm{Fe}$, although variations in the $[\mathrm{Fe} / \mathrm{H}]$ abundances are present. At present, NGC 6934 is the only Type II GC with available spectroscopy on the red sequence of the chromosome map that displays chemical variations in $\mathrm{Fe}$, but not in the $s$-elements. ${ }^{11}$ A larger sample of stars would help to assess if the Fe distribution in NGC 6934 is continuous or consistent with two discrete populations.

Another photometric similarity between NGC 6934 and anomalous GCs with chemical variations in $[s$-elements $/ \mathrm{Fe}]$ is the split SGB, as shown in Figures 1 and 8. In the anomalous GCs this feature has been associated with the presence of stellar populations with different $\mathrm{C}+\mathrm{N}+\mathrm{O}$ (Marino et al. 2011a; Marino et al. 2012a). As an example, the comparison between isochrones and the CMD of M22 reveals that a variation in $\mathrm{Fe}$

\footnotetext{
${ }^{11}$ Yong et al. (2014) have shown that the extremely Fe-rich population in M 2, in contrast to the Fe-intermediate population, does not show any enhancement in the $[s$-elements/Fe]. However, the extremely Fe-rich stars have other chemical peculiarities, such as lower $[\mathrm{Ca} / \mathrm{Fe}]$ abundances.
} 
alone could not reproduce the observed SGB split (Marino et al. 2009). In contrast, isochrones with the same age and with a metallicity difference of $0.2 \mathrm{dex}$, corresponding to the observed $\mathrm{Fe}$ difference between the normal and anomalous stars, can account for the size of the SGB split in NGC 6934, without invoking any variation in the overall $\mathrm{C}+\mathrm{N}+\mathrm{O}$. Although we cannot estimate the total $\mathrm{C}+\mathrm{N}+\mathrm{O}$ for NGC 6934 without $\mathrm{N}$-abundance measurement, the lack of additional internal variations in $s$-process elements points to no strong intra-cluster pollution by low-mass asymptotic giant branch stars that underwent third dredge-up (at least not at the level observed in the $s$ II GCs); and might in turn suggests no variations in the $[\mathrm{C}+\mathrm{N}+\mathrm{O} / \mathrm{Fe}]$ (e.g., Karakas 2010).

We emphasize here that the shape of the distribution of the anomalous-red stars in the chromosome map of NGC 6934 shows a few differences from that observed in other anomalous GCs. While most of the red stars in the anomalous GCs, such as M 22, have relatively high values of $\Delta_{C}$ F275W,F336W,F438W, suggesting an enrichment in light elements, in NGC 6934 they are located on the lower part of the diagram (see Figure 2). Even though it is probably very unlikely to have $s$-enriched stars at higher $\Delta_{C}$ F275W,F336W,F438W with a lack of them at lower values, we cannot exclude this a priori. Future observations sampling a larger number of stars on all the stellar populations appearing in the chromosome map of NGC 6934 will allow us to better constrain the extent of the $\mathrm{Fe}$ variations in this GC.

Our chemical abundance analysis suggests that there is no one-to-one correlation between the appearance of red additional sequences on the chromosome maps of Type II GCs and variations in $s$-elements and possibly $\mathrm{C}+\mathrm{N}+\mathrm{O}$. Could these sequences instead be more directly linked to enrichments in the overall metallicity? This idea opens a new perspective in the interpretation of the origin of $\sim 18 \%$ of the Milky Way GCs. Could all these objects have been significantly more massive than typical GCs in order to support a more prolonged star formation? Could the retention of SNe ejecta indicate that these objects originated in a deeper potential well, such as the nucleus of a dwarf galaxy (Da Costa 2015)? Future observations will shed light on the answers to these questions.

The authors thank the anonymous referee for a useful discussion.

Australian access to the Magellan Telescopes was supported through the National Collaborative Research Infrastructure Strategy of the Australian Federal Government.

Based on observations obtained with ESPaDOnS, located at the CFHT. CFHT is operated by the National Research Council of Canada, the Institut National des Sciences de l'Univers of the Centre National de la Recherche Scientique of France, and the University of Hawai' $i$. ESPaDOnS is a collaborative project funded by France (CNRS, MENESR, OMP, LATT), Canada (NSERC), CFHT and ESA. ESPaDOnS was remotely controlled from the Gemini Observatory, which is operated by the Association of Universities for Research in Astronomy, Inc., under a cooperative agreement with the NSF on behalf of the Gemini partnership: the National Science Foundation (United States), the National Research Council (Canada), CONICYT (Chile), Ministerio de Ciencia, Tecnología e Innovacin Productiva (Argentina) and Ministério da Ciência, Tecnologia e Inovação (Brazil).

A.F.M., G.D.C., and H.J. acknowledge support by the Australian Research Council through Discovery Early Career
Researcher Award DE160100851 and Discovery projects DP150103294 and DP150100862. A.P.M. has been supported by the European Research Council through the Starting Grant "GALFOR."

Facilities: Magellan:Clay, Gemini:Gillett, HST.

\section{ORCID iDs}

A. F. Marino (10) https://orcid.org/0000-0002-1276-5487

A. P. Milone (iD https://orcid.org/0000-0001-7506-930X

G. Piotto (i) https://orcid.org/0000-0002-9937-6387

G. Da Costa (iD https://orcid.org/0000-0001-7019-649X

M. Asplund (i) https://orcid.org/0000-0002-5804-3682

H. Jerjen (iD https://orcid.org/0000-0003-4624-9592

\section{References}

Alonso, A., Arribas, S., \& Martínez-Roger, C. 1999, A\&AS, 140, 261

Alves-Brito, A., Yong, D., Meléndez, J., Vásquez, S., \& Karakas, A. I. 2012, A\&A, 540, A3

Amarsi, A. M., Asplund, M., Collet, R., \& Leenaarts, J. 2016, MNRAS, 455,3735

Anderson, J., Sarajedini, A., Bedin, L. R., et al. 2008, AJ, 135, 2055

Bekki, K., \& Freeman, K. C. 2003, MNRAS, 346, L11

Bellazzini, M., Ibata, R. A., Chapman, S. C., et al. 2008, AJ, 136, 1147

Bergemann, M., Lind, K., Collet, R., Magic, Z., \& Asplund, M. 2012, MNRAS, 427, 27

Bernstein, R., Shectman, S. A., Gunnels, S. M., Mochnacki, S., \& Athey, A. E. 2003, Proc. SPIE, 4841, 1694

Brown, J. A., Wallerstein, G., \& Gonzalez, G. 1999, AJ, 118, 1245

Carretta, E., Bragaglia, A., Gratton, R. G., et al. 2010, ApJL, 714, L7

Carretta, E., Bragaglia, A., Gratton, R. G., et al. 2013, A\&A, 557, A138

Cassisi, S., Salaris, M., Pietrinferni, A., et al. 2008, ApJL, 672, L115

Castelli, F., \& Kurucz, R. L. 2004, arXiv:astro-ph/0405087

Chené, A.-N., Padzer, J., Barrick, G., et al. 2014, Proc. SPIE, 9151, 915147

Da Costa, G. S. 2015, IAUGA, 22, 2249201

Da Costa, G. S., Held, E. V., \& Saviane, I. 2014, MNRAS, 438, 3507

Da Costa, G. S., Held, E. V., Saviane, I., \& Gullieuszik, M. 2009, ApJ, 705,1481

D’Orazi, V., Gratton, R. G., Pancino, E., et al. 2011, A\&A, 534, A29

Dotter, A., Chaboyer, B., Jevremović, D., et al. 2008, ApJS, 178, 89

Ferraro, F. R., Dalessandro, E., Mucciarelli, A., et al. 2009, Natur, 462, 483

Gratton, R. G., Lucatello, S., Sollima, A., et al. 2013, A\&A, 549, AA41

Harris, W. E. 1996, AJ, 112, 1487

Harris, W. E. 2010, arXiv:1012.3224

Johnson, C. I., Caldwell, N., Rich, R. M., et al. 2017, ApJ, 836, 168

Johnson, C. I., \& Pilachowski, C. A. 2010, ApJ, 722, 1373

Johnson, C. I., Rich, R. M., Pilachowski, C. A., et al. 2015, AJ, 150, 63

Karakas, A. I. 2010, MNRAS, 403, 1413

Kausch, W., Noll, S., Smette, A., et al. 2015, A\&A, 576, A78

Kelson, D. D. 2003, PASP, 115, 688

Kelson, D. D., Illingworth, G. D., van Dokkum, P. G., \& Franx, M. 2000, ApJ, 531,159

Kurucz, R. L. 2009, in AIP Conf. Ser. 1171, Recent Directions in Astrophysical Quantitative Spectroscopy and Radiation Hydrodynamics: Proc. of the International Conference in Honor of Dimitri Mihalas for His Lifetime Scientific Contributions on the Occasion of His 70th Birthday. (Melville, NY: AIP), 43

Lardo, C., Pancino, E., Mucciarelli, A., et al. 2013, MNRAS, 433, 1941

Lawler, J. E., Bonvallet, G., \& Sneden, C. 2001a, ApJ, 556, 452

Lawler, J. E., Wickliffe, M. E., den Hartog, E. A., \& Sneden, C. 2001b, ApJ, 563,1075

Lee, J.-W. 2016, ApJS, 226, 16

Lind, K., Asplund, M., Barklem, P. S., \& Belyaev, A. K. 2011, A\&A, 528, A103

Lind, K., Bergemann, M., \& Asplund, M. 2012, MNRAS, 427, 50

Marino, A. F., Milone, A. P., Karakas, A. I., et al. 2015, MNRAS, 450, 815

Marino, A. F., Milone, A. P., Piotto, G., et al. 2009, A\&A, 505, 1099

Marino, A. F., Milone, A. P., Piotto, G., et al. 2011b, ApJ, 731, 64

Marino, A. F., Milone, A. P., Piotto, G., et al. 2012b, ApJ, 746, 14

Marino, A. F., Milone, A. P., Sneden, C., et al. 2012a, A\&A, 541, A15

Marino, A. F., Milone, A. P., Yong, D., et al. 2014, MNRAS, 442, 3044

Marino, A. F., Sneden, C., Kraft, R. P., et al. 2011a, A\&A, 532, A8 
Martioli, E., Teeple, D., Manset, N., et al. 2012, Proc. SPIE, 8451, 84512B

Massari, D., Mucciarelli, A., Ferraro, F. R., et al. 2014, ApJ, 795, 22

Milone, A. P., Bedin, L. R., Piotto, G., et al. 2008, ApJ, 673, 241

Milone, A. P., Marino, A. F., Piotto, G., et al. 2015, MNRAS, 447, 927

Milone, A. P., Piotto, G., Renzini, A., et al. 2017, MNRAS, 464, 3636

Norris, J. E., \& Da Costa, G. S. 1995, ApJ, 447, 680

Norris, J. E., Freeman, K. C., \& Mighell, K. J. 1996, ApJ, 462, 241

Origlia, L., Rich, R. M., Ferraro, F. R., et al. 2011, ApJL, 726, L20

Piotto, G., Milone, A. P., Bedin, L. R., et al. 2015, AJ, 149, 91

Renzini, A., D’Antona, F., Cassisi, S., et al. 2015, MNRAS, 454, 4197
Roederer, I. U., Mateo, M., Bailey, J. I., et al. 2016, MNRAS, 455, 2417

Smette, A., Sana, H., Noll, S., et al. 2015, A\&A, 576, A77

Smith, V. V., Suntzeff, N. B., Cunha, K., et al. 2000, AJ, 119, 1239

Sneden, C. 1973, ApJ, 184, 839

Suntzeff, N. B., \& Kraft, R. P. 1996, AJ, 111, 1913

Villanova, S., Geisler, D., \& Piotto, G. 2010, ApJL, 722, L18

Yong, D., \& Grundahl, F. 2008, ApJL, 672, L29

Yong, D., Grundahl, F., D’Antona, F., et al. 2009, ApJL, 695, L62

Yong, D., Grundahl, F., \& Norris, J. E. 2015, MNRAS, 446, 3319

Yong, D., Roederer, I. U., Grundahl, F., et al. 2014, MNRAS, 441, 3396 